\title{
Bioprospecting the antimicrobial, antibiofilm and antiproliferative activity of Symplocos racemosa Roxb. Bark phytoconstituents along with their biosafety evaluation and detection of antimicrobial components by GC-MS
}

Henna Sood ${ }^{1}$, Yashwant Kumar², Vipan Kumar Gupta ${ }^{3}$ and Daljit Singh Arora ${ }^{*^{*}}$ (D)

\begin{abstract}
Background: Plants provide a ray of hope to combat the ever increasing antibiotic resistance and Symplocos racemosa is a valuable medicinal plant. The study focused on highlighting the importance of this plant's phytoconstituents as potential source of novel antimicrobials against planktonic as well as biofilm forming microorganisms, along with their antiproliferative activity. The biosafety of the phytoconstituents was also established, followed by detection of probable antimicrobial components.
\end{abstract}

Methods: The best organic extractant and major groups of phytoconstituents were tested for their antimicrobial activity against reference microbial strains and drug-resistant clinical isolates. The anti-proliferative potential of the most active group of phytoconstituents was evaluated against cancerous cell lines. The in vitro biosafety of phytoconstituents was evaluated by Ames and MTT assay, while in vivo biosafety of the most active phytoconstituents, i.e., flavonoids was determined by acute oral toxicity. Further, the probable antimicrobial components in the flavonoids were detected by TLC and GC-MS.

(Continued on next page)

\footnotetext{
* Correspondence: daljit_02@yahoo.co.in

'Microbial Technology Laboratory, Department of Microbiology, Guru Nanak Dev University, Amritsar 143005, India

Full list of author information is available at the end of the article
}

(c) The Author(s). 2020 Open Access This article is licensed under a Creative Commons Attribution 4.0 International License, which permits use, sharing, adaptation, distribution and reproduction in any medium or format, as long as you give appropriate credit to the original author(s) and the source, provide a link to the Creative Commons licence, and indicate if changes were made. The images or other third party material in this article are included in the article's Creative Commons licence, unless indicated otherwise in a credit line to the material. If material is not included in the article's Creative Commons licence and your intended use is not permitted by statutory regulation or exceeds the permitted use, you will need to obtain permission directly from the copyright holder. To view a copy of this licence, visit http://creativecommons.org/licenses/by/4.0/. The Creative Commons Public Domain Dedication waiver (http://creativecommons.org/publicdomain/zero/1.0/) applies to the data made available in this article, unless otherwise stated in a credit line to the data. 
(Continued from previous page)

Results: Ethyl acetate extract was the most effective among various organic extracts, whereas phytoconstituents such as flavonoids, cardiac glycosides, saponins, tannins, triterpenes and phytosterols were the major groups present, with flavonoids being the most potent antimicrobials. The phytoconstituents displayed a significant antibiofilm potential, as exhibited by inhibition of initial cell attachment, disruption of the pre-formed biofilms and reduced metabolic activity of biofilms. The phytoconstituents were significantly active against the drug-resistant strains of E.coli, MRSA and Salmonella spp. Further, flavonoids showed significant cytotoxic effect against the cancerous cell lines but were non-cytotoxic against Vero (normal) cell line. All the test preparations were biosafe, as depicted by the Ames test and MTT assay. Also, flavonoids did not induce any abnormality in body weight, clinical signs, biochemical parameters and organs' histopathology of the Swiss albino mice during in vivo acute oral toxicity studies. The flavonoids were resolved into 4 bands (S1-S4), where S3 was the most active and its GC-MS analysis revealed the presence of a number of compounds, where Bicyclo [2.2.1] heptan-2-one,1,7,7-trimethyl-, (1S)- was the most abundant.

Conclusions: These findings suggest that the phytoconstituents from Symplocos racemosa bark could act as potential source of antimicrobial as well as antiproliferative metabolites.

Keywords: Antimicrobial, Drug resistant strains, Acute oral toxicity, Antibiofilm, Cytotoxicity, Symplocos racemosa

\section{Background}

Antibiotics have revolutionized the medical field and have helped us to live a healthy life. However, incidences of multiple resistances in human pathogenic microorganisms are on the rise and the problem has now become a global concern. The scientists are studying every aspect of antibiotic resistance [1] to come forward with possible ways out.

To meet out this objective, scientists are searching for new antimicrobials from various sources, where natural products still remain as one of the best reservoir for new compounds. The use of medicinal herbs is an age-old tradition and the recent progress in modern therapeutics has stimulated for further exploitation [2]. Numerous studies have revealed the potential of herbs as sources of drugs and have subsequently identified natural plantbased antimicrobial compounds [3-6]. The activity of these plants against different bacteria, fungi and parasites might be due to the presence of a wide variety of active secondary metabolites such as flavonoids, phenolic acids, coumarins, terpenoids and sterols [7-11].

In addition to being a potential source of novel antimicrobials, plants also play an important role in chemotherapy and represent a promising source of anticancer agents. However, the use of herbal products should be based on scientific validation, as the traditional use of any plant for medicinal purposes, by no means, guarantees the safety of the plant or treatment. Toxicity testing can identify any such risk that may be associated with herbal usage, therefore avoiding their potential short term or long term negative effects, when used as medicine [12].

One such potential medicinal plant is Symplocos racemosa Roxb. (also known as Lodhra) from the family Symplocaceae, which is an evergreen tree or shrub found in the plains and lower hills throughout North and East
India [13]. Traditionally, bark is given in menorrhagia and other uterine disorders. It is a potent remedy for inflammation and cleaning uterus [14]. Since the potential of its aqueous extract has been reported earlier in Sood et al. [15], the present study has thus been further extended to evaluate the best organic solvent and hence the major groups of bioactive phytoconstituents. The organic extract and active phytoconstituents have been tested for their various bioactivities such as antimicrobial [Post Antibiotic Effect, Minimum Inhibitory Concentration studies and Time Kill Assay; antimicrobial efficacy against drug resistant clinical isolates; anti-biofilm activity] and anti-proliferative action against the cancerous cell lines (RD, L20B, Hep2) and normal cell line (Vero). The biosafety of all the test preparations was evaluated by Ames test and MTT assay. The in vivo biosafety of the flavonoids [at a single dose $(5000 \mathrm{mg} / \mathrm{kg})$ ] has also been determined by acute oral toxicity assay using 8-10 weeks old Swiss albino mice (males and females) (Body weight: $25 \mathrm{~g}$ to $35 \mathrm{~g}$ ) [12, 16-18].

\section{Methods}

\section{Plant material}

The plant Symplocos racemosa bark was procured from a local pansari market of Ram Bagh, near Hall Gate, Amritsar, Punjab (India); which has been identified and submitted to the Herbarium of the Department of Botanical and Environmental Sciences, Guru Nanak Dev University (Amritsar), India, with an accession no. 766-768 Bot.\& Env. Sc. dated $02 / 07 / 15$. Surface sterilization of the plant material was performed as described earlier in Sood et al. [15].

\section{Test microorganisms and inoculum preparation}

All 12 reference strains of bacteria and yeast used in the study were obtained from Microbial Type Culture 
Collection (MTCC) of Institute of Microbial Technology (IMTECH), Chandigarh (India). A drug resistant isolate of MRSA, obtained from Post-Graduate Institute of Medical Education and Research (PGIMER), Chandigarh, India was also used in the study. All these cultures were preserved in the glycerol stock at $-80{ }^{\circ} \mathrm{C}$.

To add upon value to the study, some drug resistant clinical isolates were also used, which included: MRSA (DSECI 1-11), Enterococcus sp. (DSECI 12) procured from Shri Guru Ram Das medical College \& Hospital, Amritsar (Punjab), India; MDR Escherichia coli (CRIRS 112) and MDR Salmonella spp. (CRIRS 13-22) [obtained from Central Research Institute, Kasauli (H.P.)], India.

The inoculum of these organisms was prepared by $4 \mathrm{~h}$ activation in a suitable broth, followed by its standardization upto 0.5 McFarland standards [15].

\section{Organic extracts' preparation and their antimicrobial screening}

Different organic solvents such as ethyl acetate, chloroform, butanol, hexane and methanol were screened to work out the best extractant. The organic extracts were prepared by using solvent-solvent extraction of the aqueous extract (detailed in Additional file 1). The aqueous extract was prepared by suspending powdered plant material $(17.5 \mathrm{~g})$ in sterile distilled water $(100 \mathrm{~mL})$ and keeping it in hot water bath at $40^{\circ} \mathrm{C}$ for $20 \mathrm{~min}$. Hundred milliliter of filtered aqueous extract was then shaken vigorously in a separating funnel with an equal volume of a particular organic solvent, for three independent times. The pooled organic layers were concentrated in a rotavapor at $45^{\circ} \mathrm{C}$ (under vacuum). The leftover material thus obtained were dissolved in diluted Dimethyl Sulfoxide (DMSO) [(30\% (v/v)], which was then tested for their antimicrobial activity against the reference strains using the Agar Diffusion Assay (ADA).

The ADA studies were carried out as detailed in Additional file 1 . The organic extract $(0.1 \mathrm{~mL}$ of Ethyl acetate extract: $30.5 \mathrm{mg} / \mathrm{mL}$; Butanol extract: $35.42 \mathrm{mg} / \mathrm{mL}$; Hexane extract: $27.98 \mathrm{mg} / \mathrm{mL}$; Chloroform extract: $26.44 \mathrm{mg} / \mathrm{mL}$ ) was added to the wells and the plates were incubated in an upright position at $37^{\circ} \mathrm{C}$ (bacterial cultures) and $25^{\circ} \mathrm{C}$ (yeast strains) for $18-24 \mathrm{~h}$. The antimicrobial activity could be observed as a clear zone of inhibition around the wells. Any organism with inhibition zone $<12 \mathrm{~mm}$ was considered as resistant to the extract. Here, $30 \%$ DMSO served as a negative control and the experiment was performed in duplicate.

\section{Phytochemical analysis of Symplocos racemosa}

Qualitative analysis of the major groups of phytoconstituents (such as flavonoids, alkaloids, terpenoids, saponins, tannins, glycosides etc) in the powdered plant material was performed as per the standard methodology mentioned in Additional file 1. The qualitatively detected phytoconstituents were then quantified as described previously $[19,20]$ and screened for their antimicrobial potential along with some antibiotics (Additional file 1).

\section{Minimum Inhibitory Concentration (MIC) studies}

The test preparations (ethyl acetate extract, flavonoids, cardiac glycosides) were studied for their MIC against the reference microbial strains (sensitive to the respective preparations in ADA) by Agar dilution method, as per the protocol followed in Arora and Sood [21] using various concentrations (Additional file 1) prepared from their respective stock solutions [i.e., Ethyl acetate extract: $30.5 \mathrm{mg} / \mathrm{mL}$; Flavonoids: $54.75 \mathrm{mg} / \mathrm{mL}$; Cardiac glycosides: $59.5 \mathrm{mg} / \mathrm{mL}]$. Hundred microliters $(100 \mu \mathrm{L})$ of the activated test organism (adjusted to $0.5 \mathrm{McFarland}$ standards) was swabbed onto the solidified plates (containing different concentrations of a test preparation) and incubated at $37{ }^{\circ} \mathrm{C}$ (bacterial cultures) and $25^{\circ} \mathrm{C}$ (yeast strains) for $18-24 \mathrm{~h}$. The lowest concentration of the test preparations which inhibited the microbial growth were taken as their MIC. The values were compared with that of the standard antibiotics.

\section{Time kill assay and Post Antibiotic Effect (PAE)}

In order to assess the microbistatic or microbicidal nature, time kill assay of the test preparations and standard antibiotics (Gentamicin and Amphotericin B) was performed [22] at their respective stock concentrations as mentioned above. The test preparation was mixed with the $10^{-3}$ diluted inoculum of the test organism in the ratio 1:1. The inoculum containing only diluent was used as control. The mixture was then incubated at $37^{\circ} \mathrm{C}$ (bacterial cultures) and $25^{\circ} \mathrm{C}$ (yeast strains). The $100 \mu \mathrm{L}$ aliquot was plated on respective medium every $2 \mathrm{~h}$ upto $24 \mathrm{~h}$. The mean number of colonies was counted for both test and control group and the \% cell viability was calculated.

The PAE for the above mentioned test preparations were worked out according to Raja et al. [23]. The $4 \mathrm{~h}$ activated test organism was diluted 1:1000 times using suitable broth. The diluted suspension of different organisms was mixed with the test preparations [as mentioned above] in an equal ratio in a test tube and incubated for $2 \mathrm{~h}$ at $37^{\circ} \mathrm{C}$ (bacterial cultures) and $25^{\circ} \mathrm{C}$ (yeast strains) under shaking condition. Simultaneously, a control containing the diluent (without test preparation) and the respective microorganism was also set up. Following incubation, the reaction mixture was serially diluted to $10^{-3}$ using the suitable broth, whose $100 \mu \mathrm{L}$ aliquot was withdrawn at every $2 \mathrm{~h}$ interval upto $24 \mathrm{~h}$ and plated onto the medium plates. After incubation of $24 \mathrm{~h}$, the number of colonies was counted in the 
respective plates. The PAE was deduced using a formula, i.e., $\mathrm{PAE}=\mathrm{T}-\mathrm{C}(\mathrm{T}=$ time taken for number of colonies in the test to increase by $1 \log _{10} \mathrm{CFU} / \mathrm{mL} ; \mathrm{C}=$ the time required in case of untreated control).

\section{Biosafety evaluation of Symplocos racemosa bark Ames mutagenicity test}

The Ames test for different test preparations (Ethyl acetate extract: $30.5 \mathrm{mg} / \mathrm{mL}$; Flavonoids: $54.75 \mathrm{mg} / \mathrm{mL}$; Cardiac glycosides: $59.5 \mathrm{mg} / \mathrm{mL}$ ) was carried out by plate incorporation method, as described earlier [21] (for details see Additional file 1). Sodium azide was taken as a standard mutagen, while $30 \%$ DMSO were used as a negative control.

\section{MTT [3-(4, 5-dimethylthiazol-2-yl)-2, 5-diphenyl tetrazolium bromide] assay}

MTT assay was also done for the test preparations to find out their cytotoxic effect, as described earlier [24] with slight modifications (for details see Additional file $1)$. To calculate the $\%$ cell viability, the wells with untreated cells were used as control. Aliquots $(100 \mu \mathrm{L})$ of blood cells' suspension (in PBS; containing approximately $1 \times 10^{5}$ cells $/ \mathrm{mL}$ ) were dispensed into a 96 well microtitre plate in duplicates and after overnight incubation at $37^{\circ} \mathrm{C}$, the supernatant was removed carefully and $200 \mu \mathrm{L}$ of the test preparations (as mentioned in above experiments) was added and incubated further for 1 day. Following incubation, the suspension was decanted off and the cells were exposed to $20 \mu \mathrm{L}$ of MTT dye $(0.5 \%$ $\mathrm{w} / \mathrm{v})$ at $37^{\circ} \mathrm{C} / 3.5 \mathrm{~h}$, under mild shaking condition. The excess dye was pipetted out and DMSO $(50 \mu \mathrm{L})$ was added for dissolving the end product (formazan), whose absorbance was then read at $590 \mathrm{~nm}$ in a 96 wellmicrotitre plate reader and the \% viability of cells was calculated.

Since, flavonoids and cardiac glycosides showed comparatively better antimicrobial potential and were biosafe, thus, evaluated further for their antibiofilm potential and efficacy against drug resistant clinical isolates.

\section{Antibiofilm potential of the phytoconstituents of Symplocos racemosa bark}

The antibiofilm potential was tested against four organisms, i.e., Escherichia coli, Staphylococcus aureus, Klebsiella pneumoniae 1 and a yeast Candida albicans, as described earlier [25-31] and detailed in Additional File 2.

\section{Screening for biofilm formation and estimation of biofilm biomass by Crystal Violet (CV) assay}

The biofilm formation by test pathogens was screened using microtitre plate according to Stepanovic et al. [25] and Costa et al. [26], where the organisms were grown in suitable broth for $24 \mathrm{~h}$. The $200 \mu \mathrm{L}$ aliquot of the activated cultures were dispensed into microtitre plates and further incubated at for $24 \mathrm{~h}$, following which the wells were decanted off and stained with $0.1 \%$ crystal violet solution for $15 \mathrm{~min}$. The wells were destained with $150 \mu \mathrm{L}$ of $95 \%$ ethanol and the intensity of biofilm formation was affirmed in terms of Optical density (OD) of stained adherent biofilm by using an ELISA reader at $590 \mathrm{~nm}$. The results were interpreted by calculating the cut-off value (ODc), which separates biofilm producers from the non-biofilm producers, as follows:

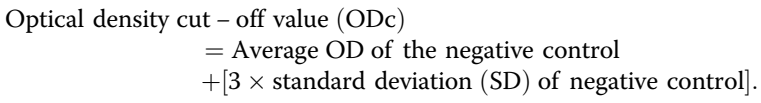

For the estimation of biofilm biomass in the succeeding assays, following the treatment of the test organisms, the culture medium from each well was discarded and plates were then washed with sterile distilled water and then dried. The plates were then stained for 15 min with a crystal violet solution $(0.1 \% \mathrm{w} / \mathrm{v})$, followed by washings with sterile distilled water. Absolute ethanol $(125 \mu \mathrm{L})$ was then added to destain the wells and the absorbance was determined at $590 \mathrm{~nm}$ to quantitatively estimate the biofilm density and the percentage inhibition was calculated using the formula as described earlier [27-29]:

\footnotetext{
Percentage inhibition $=100$

$-\left[\left\{\mathrm{OD}_{590 \mathrm{~nm}}\right.\right.$ test preparation well $/ \mathrm{OD}_{590 \mathrm{~nm}}$ negative control well without test preparation $\left.\} \times 100\right]$.
}

Upon confirmation, the following assays were carried out to establish the antibiofilm potential of the most active phytoconstituents.

\section{Inhibition of initial cell attachment}

The inhibitory potential of the partially purified phytoconstituents was carried out according to Jadhav et al. [30] and Onsare and Arora [27]. One hundred microlitres of flavonoids $(54.75 \mathrm{mg} / \mathrm{mL})$ and cardiac glycosides $(59.5 \mathrm{mg} / \mathrm{mL})$ were added to the 96-well microtitre plates, to which an equal volume of the cultures was then added. The plates were incubated at suitable temperature for $24 \mathrm{~h}$. Gentamicin and amphotericin B were used as positive control. Following incubation, the inhibition potential was established using crystal violet assay (as described above).

\section{Screening of phytoconstituents for their disruptive potential of preformed biofilms}

It was performed as described earlier [26, 27] with slight modifications. One hundred microliter aliquot of each of the $4 \mathrm{~h}$ activated cultures were dispensed into a 96-well microtitre plate and were incubated at $37^{\circ} \mathrm{C} / 24 \mathrm{~h}$ to allow biofilm formation and was treated with equal volume of the phytoconstituents, i.e., flavonoids $(54.75 \mathrm{mg} /$ 
$\mathrm{mL}$ ) and cardiac glycosides $(59.5 \mathrm{mg} / \mathrm{mL})$. The plates were further incubated at suitable temperature and biomass content was estimated after $24 \mathrm{~h}$ by the crystal violet assay.

\section{Estimation of metabolic activity by XTT assay}

Post exposure to the flavonoids, cardiac glycosides and standard antibiotics, the metabolic activity of the treated biofilms was assessed using the modified $\{2,3$-bis [2methyloxy-4-nitro-5-sulfophenyl]-2H-tetrazolium-5-carboxanilide\} (XTT) reduction assay [27, 31]. After incubation of the biofilm with the test preparations for $24 \mathrm{~h}$, menadione-XTT mixture was then added to each well, followed by $2 \mathrm{~h}$ incubation at the suitable temperature (under dark conditions). The quantification of color was then done using microtitre plate reader (Bio-Rad 680$\mathrm{XR}$, Tokyo, Japan) at $490 \mathrm{~nm}$. The mean absorbance of test wells was determined in comparison to that of negative control.

\section{Antimicrobial potential of the phytoconstituents against drug- resistant clinical isolates of MRSA, Escherichia coli, Enterococcus sp. and Salmonella spp.}

The flavonoids $(54.75 \mathrm{mg} / \mathrm{mL})$ and cardiac glycosides $(59.5$ $\mathrm{mg} / \mathrm{mL}$ ) were also tested for their antimicrobial efficacy against clinical isolates of drug resistant strains (Table T1 of Additional File 5) of Escherichia coli, Salmonella spp., MRSA and Enterococcus sp. by Agar Diffusion Assay (Additional file 2). The sensitivity of different organisms was measured in terms of inhibition zone (in $\mathrm{mm}$ ). The MIC of the most sensitive organisms was also worked out by broth dilution method [32]. Different concentrations of each phytoconstituent $(0.09 \mathrm{mg} / \mathrm{mL}-50 \mathrm{mg} / \mathrm{mL})$ were made from their respective stock and mixed with $50 \mu \mathrm{L}$ of actively growing bacterial suspension in a 96 well microtitre plate. The inoculum without the test compound served as a negative control and the plates were incubated at $37^{\circ} \mathrm{C}$ for $24 \mathrm{~h}$. The lowest concentration with no visible growth was defined as the Minimum Inhibitory Concentration (MIC). To determine the MBC, the aliquots from the wells showing no visible growth were swabbed further onto the nutrient agar plates and incubated at $37^{\circ} \mathrm{C}$ for $24 \mathrm{~h}$. The concentration corresponding to no growth on the plates was taken as MBC.

The above experiments revealed the flavonoids to possess a better and significant bioactivity in comparison to cardiac glycosides, hence, were pursued further to evaluate their anti-proliferative potential.

In vitro cytotoxicity against cancerous cell lines, i.e., RD (human Rhabdomyosarcoma), Hep2 (human epithelioma of larynx) and L20B (diploid mouse lung cell line)

The flavonoids was studied for their activity against the three cell lines obtained from Central Research Institute (C.R.I), Kasauli (H.P.), India by MTT [3-(4,5- dimethylthiazol-2-yl)-2,5-diphenyl tetrazolium bromide] assay as described previously [33-35] with slight modifications. The cytotoxic effect was compared to a normal cell line, i.e., Vero (African Green monkey kidney normal cell) line. The experiment was performed in triplicates as per protocol detailed in Additional file 3. Two fold serial dilutions $(10 \mathrm{mg} / \mathrm{mL}$ to $0.039 \mathrm{mg} / \mathrm{mL})$ were prepared from a stock $(10 \mathrm{mg} / \mathrm{mL})$, where the untreated cells (containing only diluent, i.e., 30\% DMSO) were taken as control. The percent growth inhibition was calculated with respect to untreated control. The $\mathrm{IC}_{50}$ of the flavonoids against the tested cancerous cell lines was deduced from the resultant graphs obtained.

The flavonoids were the most effective phytoconstituents, as evident from their significant antimicrobial, antibiofilm and antiproferative potential and were biosafe as revealed by in vitro assays. Therefore, to re-ensure their biosafety and consumability, they were subjected to in vivo acute oral toxicity study.

\section{In vivo acute oral toxicity studies of flavonoids}

The in vitro biosafety of the flavonoids was re-confirmed under in vivo conditions using an animal model (Swiss albino mice), as per the experimental details mentioned earlier [12, 16], with slight modifications, which has been detailed in Additional file 4. Total 24 healthy Swiss albino mice (males and females) (25-35 g body weight and 8-10 weeks old), were procured through proper channel from the Animal Breeding Facility of the Animal house of Central Research Institute, Kasauli, Himachal Pradesh, India. The mice were randomly divided into 2 test groups (6- male; 6- female) and 2 control groups (6male; 6 - female), where the test groups were exposed to a single dose $(5000 \mathrm{mg} / \mathrm{kg})$ of the flavonoids by oral route. This study was carried out in accordance with the principles of the Basel Declaration and recommendations of Organization of Economic Co-operation and Development (OECD) guideline 420. The study was carried out at Central Research Institute, Kasauli and the protocol was approved by the Institutional Animal Ethics Committee (IAEC) of Central Research Institute (C.R.I), Kasauli (H.P.), India; working under the guidance of Committee for the Purpose of Control and Supervision of Experiments on Animals (CPCSEA), New Delhi, India (No. CPCSEA/IAEC/CRI/14-114-2016). On the 15th day, the final weight of mice was noted and were anesthetized in the laboratory using a mixture of xylaxine and ketamine (at a concentration of $5 \mathrm{mg} / \mathrm{kg}$ b.wt. and $2.5 \mathrm{mg} / \mathrm{kg}$ b.wt., respectively). The blood samples were collected and the separated serum was used to determine the Liver functioning (Bilirubin content, ALT, AST, ALP) as well as Kidney functioning parameters (Creatinine, Urea). All the unconscious animals were then subjected to euthanasia by an overdose of 
anesthesia (mixture of xylaxine and ketamine at a concentration of $5 \mathrm{mg} / \mathrm{kg}$ and $2.5 \mathrm{mg} / \mathrm{kg}$ b.wt., respectively), as anesthetic overdose is the most commonly used method for euthanasia of mice because of being a simple, aesthetic and least painful method, with a lower blood loss associated with euthanasia [36]. Following euthanasia, the liver, kidney and heart from all the mice were extracted and prepared for the histopathological analysis.

\section{Detection of the probable bioactive components of the flavonoids}

In order to find out the component responsible for antimicrobial activity of the most active phytoconstituent, i.e., flavonoids, various techniques such as TLC, PreparativeTLC and Gas Chromatography-Mass Spectrometry (GCMS) analysis were performed.

\section{Thin Layer Chromatography (TLC) analysis}

To work out the best solvent system, the quantitatively isolated flavonoids were subjected to TLC on the precoated Silica gel $\mathrm{F}_{254}$ plates using different solvent systems. The developed chromatograms were allowed to air dry and were visualized using natural light, UV light $(254 \mathrm{~nm}, 365 \mathrm{~nm})$ and iodine vapors so as to assess the degree of separation of bands and the Retention factor $\left(R_{\mathrm{f}}\right)$ were calculated for the bands resolved in the most suitable solvent system. The best worked out solvent system was taken up for quantitatively separating the bands using Preparative TLC (P-TLC) method.

\section{Quantitative separation of the bands using Preparative TLC} ( $P$-TLC) method and their antimicrobial screening

The bands were resolved and separated on Silica gel $F_{254}$ using the selected combination of extraction solvents. Once developed, the separated bands were scrapped off carefully into separate vials, which were eluted overnight in methanol. The solvent containing the eluted band was decanted off carefully, which was evaporated to dryness to obtain a constant weight. The separated bands were dissolved in a minimum known volume of methanol and were screened for their antimicrobial activity against Staphylococcus aureus, Staphylococcus epidermidis (Gram positive); Klebsiella pneumoniae 1, Shigella flexneri (Gram negative) and a yeast Candida albicans using Disc diffusion method, where the filter paper discs were impregnated with $20 \mu \mathrm{L}$ of this suspension, while methanol alone acted as a negative control. The band/s showing the most significant antimicrobial activity was then subjected to GC-MS analysis.

\section{Gas Chromatography-Mass Spectrometry (GC-MS) analysis} The GC-MS analysis of the most active band/s was carried out using Thermo Trace 1300GC coupled with
Thermo TSQ 800 Triple Quadrupole MS with column BP 5MS $(30 \mathrm{~m} \mathrm{X} 0.25 \mathrm{~mm}, 0.25 \mu \mathrm{m})$. The instrument was set to an initial temperature of $60^{\circ} \mathrm{C}$ and maintained at this temperature for $3 \mathrm{~min}$. At the end of this period the oven temperature was raised to $280^{\circ} \mathrm{C}$, at an increase rate of $15{ }^{\circ} \mathrm{C} / \mathrm{min}$ and maintained for $19 \mathrm{~min}$. Injection port temperature was ensured as $260^{\circ} \mathrm{C}$ and Helium flow rate as $1.2 \mathrm{~mL} / \mathrm{min}$. The ionization voltage was $70 \mathrm{eV}$. The injector was used with S (split) mode, with a split ratio of 30:1 and the injection volume of samples was $1 \mu \mathrm{L}(1 \mathrm{mg} / \mathrm{mL})$. Mass spectral scan range was set at $50-650(\mathrm{~m} / \mathrm{z})$. Using computer searches on a NIST Ver. 2.0 MS data library and by comparing the spectrum obtained through GC-MS, compounds probably present in the flavonoids were identified.

\section{Data analysis}

The statistical analysis was done by One way ANOVA followed by Tukey's t-test at 95\% level of confidence using IBM SPSS Statistics Data editor Version 20 in case of acute oral toxicity study, antimicrobial activity of organic extracts and antimicrobial evaluation against MDR clinical isolates. Standard error of means (SEM) was applied to Table 2, 3, 4, 5 and 6 using Microsoft excel, 2010 and $\mathrm{IC}_{50}$ in the in vitro cytotoxicity assay was calculated using Microsoft excel, 2010.

\section{Results \\ Organic extracts' preparation and their antimicrobial screening}

To find out the best organic solvent for effective leaching out of the active components, various solvents such as ethyl acetate, butanol, chloroform, hexane and methanol were used, where methanol was miscible with the aqueous extract of Symplocos racemosa. Among all the organic extracts tested, the ethyl acetate extract demonstrated the highest activity against 10 out of 13 test organisms, with an average inhibition zone (IZ) of 14.92 $\mathrm{mm}$, where Staphylococcus aureus (Gram positive), Klebsiella pneumoniae 1 (Gram negative) and Candida albicans (Yeast) were the most sensitive organisms with an IZ of $19.5 \mathrm{~mm}, 23 \mathrm{~mm}$ and $21 \mathrm{~mm}$, respectively (Fig. A6 in Additional File 5). Butanolic extract followed up next and was active against 9 out of 13 strains (average $\mathrm{IZ}=$ $10.92 \mathrm{~mm}$ against all the tested organisms). Here, Staphylococcus aureus was the most sensitive organism (19 mm). The hexane extract was active against Candida albicans and Klebsiella pneumoniae 1, while the chloroform extract was completely inactive against all the tested microorganisms. The efficacy of ethyl acetate extract and the butanolic extract did not differ significantly from each other $(p>0.05)$, but ethyl acetate had a higher average IZ against the test organisms, hence was considered as the best organic extractant. The efficacy of both 
the extracts differed significantly from that of hexane extract of Symplocos racemosa $(p \leq 0.05)$ at 5\% level of significance, as revealed by One way ANOVA followed by Post hoc Tukey's t-test. Enterococcus faecalis, Klebsiella pneumoniae 2 and Candida tropicalis, however, remained resistant to all the organic extracts of Symplocos racemosa.

\section{Qualitative and quantitative phytochemical evaluation}

Major phytochemical groups such as flavonoids, cardiac glycosides, saponins, tannins, triterpenes and phytosterols were detected (Table 1); however, alkaloids, diterpenes and coumarins were absent. Among the isolated phytoconstituents, cardiac glycosides were the most abundant, i.e., $50.25 \% / g$ plant powder, followed by flavonoids $(10.39 \% / \mathrm{g})$, while triterpenes $(1.193 \% / \mathrm{g})$ were present in least amount (Fig. A1 in Additional file 5).

Among all the isolated phytoconstituents, flavonoids were the most effective (Table 2) with inhibition zone ranging from $16.6 \mathrm{~mm}$ to $25.66 \mathrm{~mm}$, but were ineffective against Enterococcus faecalis. However, Candida albicans $(25.66 \mathrm{~mm})$ and Candida tropicalis $(17.3 \mathrm{~mm})$ were quite sensitive to it. Cardiac glycosides showed an inhibition zone ranging from $12.3 \mathrm{~mm}$ to $25.66 \mathrm{~mm}$, where Klebsiella pneumoniae 1 showed maximum susceptibility. Salmonella Typhimurium 1, Escherichia coli, Shigella flexneri and yeast Candida tropicalis were resistant to it. Saponins were effective only against Klebsiella pneumoniae 1 and Candida albicans. However, triterpenes, tannins and phytosterols were completely inactive against all the test microorganisms. The antimicrobial efficacy of flavonoids was comparable to both the antibiotics (gentamicin and chloramphenicol) in case of Staphylococcus epidermidis, whereas it was comparable to chloramphenicol in case of Escherichia coli, Klebsiella pneumoniae 2 and Pseudomonas aeruginosa.

Minimum Inhibitory Concentration (MIC)

MIC of the ethyl acetate extract, flavonoids and cardiac glycosides was quite variable (Table 3). Ethyl acetate

Table 1 Qualitative phytochemical analysis of Symplocos racemosa bark

\begin{tabular}{|c|c|c|c|}
\hline Phytoconstituents & Detected group & Stock solution $(\mathrm{mg} / \mathrm{mL})$ & Antimicrobial activity \\
\hline Alkaloids & & NA & NA \\
\hline Mayer's reagent test & $-a^{a}$ & & \\
\hline Hager's reagent test & - & & \\
\hline Wagner reagent test & - & & \\
\hline Flavonoids & & 54.75 & $+++c$ \\
\hline Shinoda test (Magnesium turnings) & $t^{\mathrm{b}}$ & & \\
\hline Zinc-hydrochloride reduction test & + & & \\
\hline Lead acetate test & + & & \\
\hline Ferric chloride reagent test & - & & \\
\hline Saponins & & 41.6 & $+{ }^{d}$ \\
\hline Froth test & + & & \\
\hline Tannins & & 100.5 & + \\
\hline Ferric chloride reagent test & + & & \\
\hline Lead acetate test & + & & \\
\hline Cardiac glycosides & & 59.5 & $++^{e}$ \\
\hline Keller- killiani test & + & & \\
\hline \multicolumn{4}{|l|}{ Terpenoids } \\
\hline Triterpenes (Salkowski's test) & + & 12 & $-f$ \\
\hline Diterpenes (Copper acetate test) & - & NA & NA \\
\hline Anthranol glycosides & & ND & ND \\
\hline Borntrager's test & + & & \\
\hline Phytosterols & & 38 & - \\
\hline Libermann Burchard's test & + & & \\
\hline Salkowski's test & + & & \\
\hline Coumarins & - & ND & - \\
\hline
\end{tabular}

${ }^{\text {a }}$ - absent; ${ }^{\text {b}}$ - present; ${ }^{c}$ - most active; ${ }^{d}$ - least active; ${ }^{\text {e- }}$ active; ${ }^{f}$ - not active NA- Not applicable, ND- Not done 
Table 2 Antimicrobial activity of phytoconstituents isolated from Symplocos racemosa bark

\begin{tabular}{|c|c|c|c|c|c|}
\hline \multirow{2}{*}{$\begin{array}{l}\text { Test } \\
\text { Organisms }\end{array}$} & \multicolumn{5}{|c|}{ Average zone of inhibition $(\mathrm{mm})^{* *}$} \\
\hline & Flavonoids & Saponins & Cardiac Glycosides & Gentamicin $^{*}$ & Chloramphenicol $^{\mp}$ \\
\hline SA & $19.66 \pm 0.333$ & $-*$ & $15.66 \pm 0.333$ & $34.5 \pm 0.50$ & $26 \pm 1.00$ \\
\hline SE & $22.33 \pm 0.333$ & - & $16.33 \pm 0.333$ & $26.5 \pm 0.50$ & $28.5 \pm 0.50$ \\
\hline EC & $23 \pm 1$ & - & - & $31 \pm 1.00$ & $25 \pm 0$ \\
\hline EF & - & - & $12.33 \pm 0.333$ & $27.5 \pm 0.50$ & $26.5 \pm 0.50$ \\
\hline KP1 & $20.33 \pm 0.333$ & $14.33 \pm 0.333$ & $25.66 \pm 0.333$ & $40.5 \pm 0.50$ & $38 \pm 1.00$ \\
\hline KP2 & $25.66 \pm 0.333$ & - & $19.33 \pm 0.333$ & $37.5 \pm 1.50$ & $26.5 \pm 0.50$ \\
\hline SF & $16.66 \pm 0.333$ & - & - & $30.5 \pm 0.50$ & $27.5 \pm 0.50$ \\
\hline ST1 & $16 \pm 0$ & - & - & $35 \pm 0$ & $23 \pm 0$ \\
\hline ST2 & $24 \pm 0.577$ & - & $17 \pm 0$ & $43 \pm 1.00$ & $40.5 \pm 0.50$ \\
\hline PA & $21.66 \pm 0.333$ & - & $15.66 \pm 0.333$ & $40.5 \pm 0.50$ & $28.5 \pm 0.50$ \\
\hline $\mathrm{CA}$ & $25.66 \pm 0.333$ & $15.33 \pm 0.333$ & $25 \pm 0$ & $36.5 \pm 0.50^{\mathrm{a}}$ & ND \\
\hline $\mathrm{CT}$ & $17.33 \pm 0.333$ & - & - & $27.5 \pm 0.50^{\mathrm{a}}$ & ND \\
\hline MRSA & $24.33 \pm 0.666$ & - & $17.66 \pm 0.333$ & $42 \pm 0$ & $39.5 \pm 0.50$ \\
\hline
\end{tabular}

Concentrations used: Flavonoids- $54.75 \mathrm{mg} / \mathrm{mL}$; Saponins- $41.6 \mathrm{mg} / \mathrm{mL}$; Cardiac glycosides- $59.5 \mathrm{mg} / \mathrm{mL} ;{ }^{¥}$ Concentration used: $1 \mathrm{mg} / \mathrm{mL}$

${ }^{*}$ No activity; ${ }^{* *}$ Values are expressed as Mean \pm SEM of three determinations; $N D$ - not done

${ }^{a}$ Amphotericin B (1 mg/mL); ${ }^{b}$ Organisms- SA- Staphylococcus aureus (MTCC 740); SE- Staphylococcus epidermidis (MTCC 435); EC- Escherichia coli (MTCC 119); EFEnterococcus faecalis (MTCC 439); KP1- Klebsiella pneumoniae 1 (MTCC 109); KP2- Klebsiella pneumoniae 2 (MTCC 530); SF- Shigella flexneri (MTCC 1457); ST1-

Salmonella Typhimurium 1 (MTCC 98); ST2- Salmonella Typhimurium 2 (MTCC 1251); PA- Pseudomonas aeruginosa (MTCC 741); CA- Candida albicans (MTCC 227);

CT- Candida tropicalis (MTCC 230); MRSA- Methicillin- Resistant Staphylococcus aureus

extract was found to be highly potent (with MIC range of $0.5-3 \mathrm{mg} / \mathrm{mL}$ ), in comparison to the phytoconstituents (Flavonoids and Cardiac glycosides), with the MIC values being lowest against Salmonella Typhimurium 2 and Pseudomonas aeruginosa. Further, the MIC for cardiac glycosides and flavonoids ranged from $0.5-10 \mathrm{mg} /$ $\mathrm{mL}$ and $0.7-10 \mathrm{mg} / \mathrm{mL}$, respectively, where Klebsiella pneumoniae 1 and Candida albicans were the most sensitive organisms showing the lowest MIC values. For Pseudomonas aeruginosa and MRSA, cardiac glycosides showed a higher MIC $(10 \mathrm{mg} / \mathrm{mL}$ and $5 \mathrm{mg} /$ $\mathrm{mL}$, respectively) than the ethyl acetate extract $(0.5-$ $0.7 \mathrm{mg} / \mathrm{mL})$ and flavonoids $(1 \mathrm{mg} / \mathrm{mL})$. The MIC values obtained for ethyl acetate extract and flavonoids were comparable to that of chloramphenicol against Pseudomonas aeruginosa.

Table 3 Minimum Inhibitory Concentration (MIC) of the various test preparations of Symplocos racemosa bark

\begin{tabular}{|c|c|c|c|c|c|}
\hline \multirow[t]{2}{*}{ Organisms $^{d}$} & \multicolumn{5}{|c|}{ MIC (mg/mL) } \\
\hline & $\overline{E A^{b}}$ & Flavonoids & Cardiac glycosides & Gentamicin & Chloramphenicol \\
\hline SA & 0.7 & 3 & 1 & 0.0002 & 0.01 \\
\hline SE & 1 & 3 & 1 & 0.01 & 0.01 \\
\hline $\mathrm{EC}$ & 1 & 3 & ND & 0.005 & 0.01 \\
\hline $\mathrm{EF}$ & $N D^{a}$ & ND & ND & 0.03 & 0.3 \\
\hline KP1 & 0.5 & 0.7 & 0.5 & 0.0002 & 0.01 \\
\hline KP2 & ND & 1 & 10 & 0.0005 & 0.001 \\
\hline SF & 1 & 3 & ND & 0.005 & 0.01 \\
\hline ST1 & 3 & 1 & ND & 0.005 & 0.1 \\
\hline ST2 & 0.5 & 3 & 3 & 0.0003 & 0.001 \\
\hline PA & 0.5 & 0.7 & 10 & 0.005 & 0.7 \\
\hline $\mathrm{CA}$ & 0.7 & 0.7 & 0.5 & $0.0003^{c}$ & ND \\
\hline $\mathrm{CT}$ & ND & 10 & ND & $0.1^{c}$ & ND \\
\hline MRSA & 1 & 1 & 5 & 0.005 & 0.01 \\
\hline
\end{tabular}

${ }^{\mathrm{a}}$ Not determined; ${ }^{\mathrm{b}}$ Ethyl acetate extract; ${ }^{\mathrm{C}}$ Amphotericin B ${ }^{\mathrm{d}}$; SA- Staphylococcus aureus; SE- Staphylococcus epidermidis; EC- Escherichia coli; KP1- Klebsiella pneumoniae 1; KP2- Klebsiella pneumoniae 2; SF- Shigella flexneri; ST1- Salmonella Typhimurium 1; ST2- Salmonella Typhimurium 2; PA- Pseudomonas aeruginosa; CA- Candida albicans; CT- Candida tropicalis; MRSA- Methicillin- Resistant Staphylococcus aureus 


\section{Time kill assay and Post Antibiotic Effect (PAE)}

A variable killing time was observed for different test preparations (ethyl acetate extract, flavonoids and cardiac glycosides) against different test pathogens (Fig. A2 in Additional file 5). Pseudomonas aeruginosa and Candida albicans were killed instantaneously upon incubation with ethyl acetate extract, whereas Salmonella Typhimurium 1 took a maximum of $24 \mathrm{~h}$ incubation period to achieve complete killing. In case of Shigella flexneri and MRSA, the killing time ( $2 \mathrm{~h}$ and $6 \mathrm{~h}$, respectively) was similar to that of standard antibiotic gentamicin. Flavonoids were also quite effective against the test organisms, where Candida albicans was killed instantaneously. Escherichia coli and Pseudomonas aeruginosa were killed completely in $2 \mathrm{~h}$, whereas Salmonella Typhimurium 1 and Salmonella Typhimurium 2 took 6 $\mathrm{h}$ for complete killing. Their Killing time was lesser than Gentamicin in case of organisms like Staphylococcus aureus, Klebsiella pneumoniae 2, Salmonella Typhimurium 2, Pseudomonas aeruginosa and Candida albicans. Cardiac glycosides had a kill time falling between 2 and $8 \mathrm{~h}$, where $2 \mathrm{~h}$ incubation was sufficient to kill Klebsiella pneumoniae 1 and Candida albicans. They were better in case of organisms like Klebsiella pneumoniae 1 and Klebsiella pneumoniae 2. Flavonoids were more efficient in killing majority of the organisms than the ethyl acetate extract and cardiac glycosides, as evident from their lesser killing time. All the test preparations (ethyl acetate extract, flavonoids and cardiac glycosides) were highly effective against Escherichia coli $(2 \mathrm{~h})$, Pseudomonas aeruginosa $(0-4 \mathrm{~h})$ and Candida albicans $(0-2 \mathrm{~h})$.

Further, Flavonoids and cardiac glycosides showed a Post Antibiotic Effect (PAE) of 2-4 h and 2-6 h, respectively (Fig. A3 in Additional file 5). Flavonoids were equally effective $(4 \mathrm{~h})$ against Salmonella Typhimurium 2 and Klebsiella pneumoniae 2, whereas cardiac glycosides had a maximum effect against Candida albicans (6h). The ethyl acetate extract also showed a similar PAE of 2-6h. Among all the test preparations (ethyl acetate extract, flavonoids and cardiac glycosides), the longest effectivity was observed against Candida albicans (4-6 h), whereas for organisms like Staphylococcus aureus, Salmonella Typhimurium 2 and Klebsiella pneumoniae 2, their effect lasted for 2-4 h (Fig. A3 in Additional file 5).

\section{Biosafety evaluation}

All the test preparations, i.e., ethyl acetate extract, flavonoids and cardiac glycosides were found to be noncytotoxic and non-mutagenic in nature, as assessed by Ames test and MTT assay, respectively (Table T2 in Additional file 5). In Ames test, no revertant colonies were seen upon exposure to the test preparations as compared to the numerous revertant colonies (856) obtained upon exposure to the known mutagen, i.e., sodium azide. In MTT assay, the test preparations were found to be non-cytotoxic as evident from the high viability of the blood cells post-exposure [96.02\% (ethyl acetate extract); 91.83\% (flavonoids); 96.56\% (cardiac glycosides)].

\section{Antibiofilm potential of the phytoconstituents isolated from Symplocos racemosa bark}

The most active phytoconstituents, i.e., flavonoids and cardiac glycosides were assessed for their antibiofilm potential against the test pathogens. The Optical Density cut-off value (ODc) of the negative control was 0.097. All the test organisms were found to be strong biofilmformers as their average OD values were higher than $4 \times$ ODc (0.388), viz., 0.649 (Klebsiella pneumoniae 1), 0.720 (Staphylococcus aureus), 0.892 (Candida albicans) and 0.557 (Escherichia coli).

\section{Inhibition of initial cell attachment}

The flavonoids and cardiac glycosides effectively inhibited the attachment of the planktonic cells of these organisms (Fig.1a). Flavonoids (\% Inhibition: 61.01$69.33 \%$ ) were found to be more active than cardiac glycosides (\% Inhibition: 61.64-66.66\%) against the tested organisms. The maximum \% inhibition was seen against Candida albicans and Klebsiella pneumoniae 1. The inhibitory potential of phytoconstituents was in close proximity to the respective antibiotics (gentamicin and amphotericin B) (Fig.1a). This inhibitory effect was statistically compared, where the inhibitory potential of the phytoconstituents and the standard antibiotics was statistically different $(p \leq 0.05)$ against all the four test organisms, as revealed by One way ANOVA followed by Tukey's t-test. The individual effect of phytoconstituents and antibiotics on the four test organisms was statistically variable and significant, however, the inhibitory effect of flavonoids and cardiac glycosides against $C$. albicans and $K$. pneumoniae 1; antibiotic (gentamicin) against $S$. aureus and $K$. pneumoniae 1 did not show any statistical difference ( $p \geq 0.05)$ (Fig. 1a).

\section{Disruptive potential on the pre-formed biofilms}

The test preparations exhibited varying efficacy towards the pre-formed biofilms of the test organisms (Fig. 1b). Flavonoids were more effective to disrupt the preformed biofilms than the cardiac glycosides. In case of Candida albicans, the inhibitory potential of flavonoids (61.85\%) and cardiac glycosides (57.73\%) was comparable to amphotericin B (65.97\%), though the values showed statistically significant difference $(\mathrm{p} \leq 0.05)$. The inhibitory potential of the phytoconstituents and the standard antibiotics was statistically different $(\mathrm{p} \leq 0.05)$ against all the four test organisms, as revealed by One 


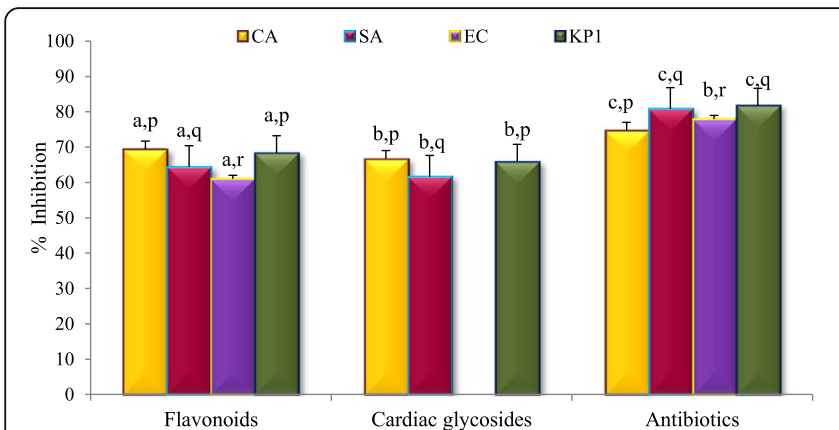

(a)

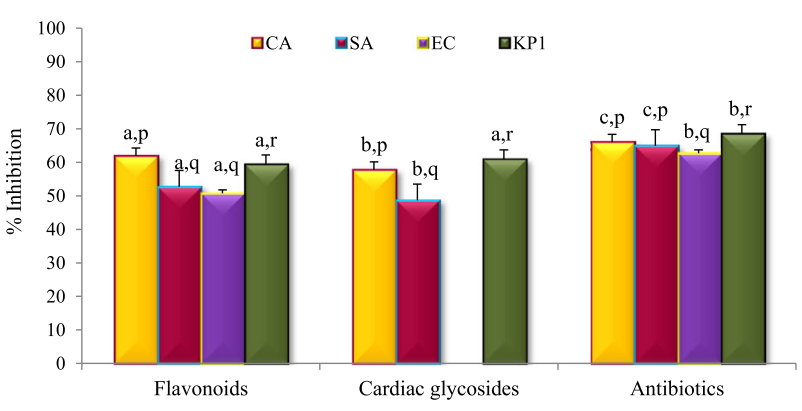

(b)

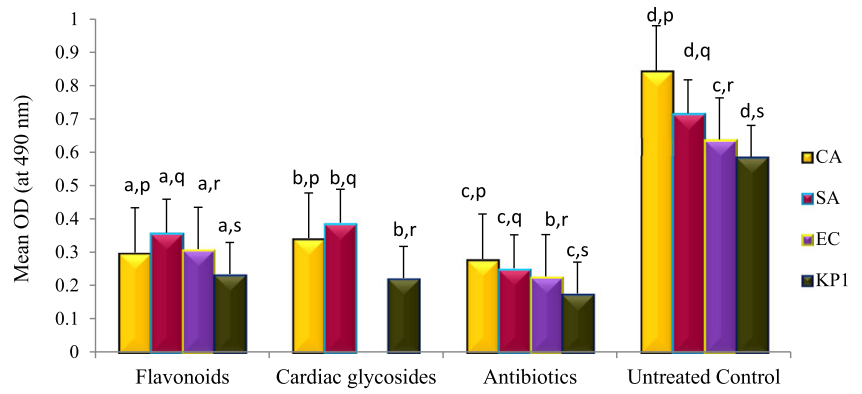

(c)

Fig. 1 Inhibitory action of the Partially Purified Phytoconstituents of Symplocos racemosa bark on the a Initial cell attachment $\mathbf{b}$ Pre-formed biofilms of the test organisms c Metabolic activity of the biofilms.. EC-Escherichia coli; KP1- Klebsiella pneumoniae 1, CA- Candida albicans, SAStaphylococcus aureus. Similar alphabetic letters on the bar columns before and after the comma represent no significant difference at $95 \%$ confidence level among different treatments for each organism and among test organisms for each treatment, respectively as indicated by Oneway ANOVA followed by Tukey's t-test

way ANOVA followed by Tukey's t-test, except for the inhibitory effect of flavonoids and cardiac glycosides against K. pneumoniae 1 . The individual effect of phytoconstituents and antibiotics on the four test organisms was statistically variable and significant, however, the inhibitory effect of flavonoids against $S$. aureus and E. coli; antibiotic (gentamicin) against $S$. aureus and $C$. albicans did not show any statistical difference ( $\mathrm{p} \geq 0.05)$ (Fig. 1b).

\section{Metabolic activity of the treated pre-formed biofilms by using XTT assay}

The flavonoids and cardiac glycosides considerably reduced the cell viability, as indicated by the reduced metabolic activity (low mean absorbance values) (Fig. 1c). In case of Candida albicans, flavonoids (mean absorbance $=$ 0.298 ) were more effective than cardiac glycosides (mean absorbance $=0.342$ ), however, both of them had an effect comparable to amphotericin B (mean absorbance $=0.279$ ). Cardiac glycosides reduced the metabolic activity of Klebsiella pneumoniae 1 biofilm more efficiently than the flavonoids, whereas, the effect of flavonoids and cardiac glycosides was almost similar in case of Staphylococcus aureus. These were found to be good biofilms inhibitors by efficiently reducing the metabolically active cells. It was observed that the inhibitory potential of the phytoconstituents and the standard antibiotics was statistically different $(p \leq 0.05)$ from each other against all the four test organisms, as revealed by One way ANOVA followed by Tukey's t-test. Also, the individual effect of phytoconstituents and antibiotics on the four test organisms showed statistically significant difference $(p \leq 0.05)$.

\section{Antimicrobial potential of the phytoconstituents against the resistant clinical isolates}

The most active phytoconstituents, i.e., flavonoids and cardiac glycosides, when tested against the 34 drug resistant clinical isolates of E. coli and Salmonella spp. (CRIRS1-22) (Group 1) as well as MRSA (DSECI01-12) (Group 2) and an Enterococcus sp., showed a significant antimicrobial potential with overall Inhibition Zone (IZ) ranging from $12.33-28.33 \mathrm{~mm}$ (Table 4). Among the two phytoconstituents, flavonoids showed the highest and broad spectrum antimicrobial activity against the 33 out of total 34 clinical isolates, with IZ ranging from 14.66$28.33 \mathrm{~mm}$, while Cardiac glycosides were active only against 14 out of total 34 clinical isolates with an IZ range of $12.33-17.33 \mathrm{~mm}$. This difference in the antimicrobial efficacy of the two phytoconstituents was found to be 
statistically significant $(p \leq 0.05)$, as revealed by one way ANOVA followed by Post hoc Tukey's t-test (Table 4).

In case of Group 1 (CRIRS1-22), i.e., clinical isolates of E. coli and Salmonella spp., flavonoids showed a broad spectrum potential against all the 22 strains (14.66-22.66 $\mathrm{mm}$ ) belonging to this group (Table 4), with an average Inhibition Zone (IZ) of $17.05 \mathrm{~mm}$. Cardiac glycosides were active against only 6 organisms (CRIRS $4,5,6,8,10,13$ ) out of 22 strains, with an overall average IZ of $3.72 \mathrm{~mm}$ (Table 4). Hence, the flavonoids were more effective than the cardiac glycosides, since the difference in the average Inhibition Zone (IZ) of the two phytoconstituents against Group 1 (CRIRS1-22) isolates was found to be statistically significant $(p \leq 0.05)$, as revealed by one way ANOVA followed by Post hoc Tukey's t-test.

Further, Flavonoids were significantly effective against 10 out of 11 strains of MRSA (DSECI 01-11) (Group 2), with IZ ranging from 20 to $28.33 \mathrm{~mm}$, giving an average IZ of $22.30 \mathrm{~mm}$. Here, DSECI 04 was the most sensitive $(28.33 \mathrm{~mm})$, while DSECI 11 was completely resistant. Cardiac glycosides were effective against 8 out of 12 strains, where the IZ ranged from 14 to $18 \mathrm{~mm}$; hence giving an average IZ of $9.96 \mathrm{~mm}$. DSECI04 was the most sensitive $(18 \mathrm{~mm})$, whereas the strains DSECI07, DSEC I09, DSECI10 and DSECI11 were completely resistant. Hence, flavonoids showed a higher antimicrobial effectiveness than the cardiac glycosides, since the difference in the average Inhibition Zone (IZ) of the two phytoconstituents against DSECI 01-11) (Group 2) isolates was found to be statistically significant $(\mathrm{p} \leq 0.05)$, as revealed by one way ANOVA followed by Post hoc Tukey's t-test.

The Enterococcus sp. (DSECI 12) was susceptible to both flavonoids and cardiac glycosides, where flavonoids were more active $(23.33 \mathrm{~mm})$ than cardiac glycosides $(15.66 \mathrm{~mm})$ and this difference was statistically significant $(p \leq 0.05)$. Because of their effectiveness, the flavonoids were further tested against the 9 most susceptible strains (CRIRS7, CRIRS8, CRIRS13, CRIR S17, DSECI03, DSECI04, DSECI07, DSECI09 and DSECI10) for their MIC and MBC. The MIC values ranged from 15 to $35 \mathrm{mg} / \mathrm{mL}$, lowest being against CRIRS7, DSECI07 and DSECI10 $(15 \mathrm{mg} / \mathrm{mL})$. The corresponding $\mathrm{MBC}$ values ranged from 20 to $40 \mathrm{mg} /$ $\mathrm{mL}$ (Table T3 in Additional file 5).

\section{In vitro cytotoxicity studies by MTT assay against RD, L20B and Hep2 cell lines}

The Flavonoids were further evaluated for their cytotoxicity against some cancerous cell lines, where a cytotoxic effect was seen with $\mathrm{IC}_{50}$ ranging from 361 to $494 \mu \mathrm{g} /$ $\mathrm{mL}$. The lowest $\mathrm{IC}_{50}(361 \mu \mathrm{g} / \mathrm{mL})$ was seen against Hep2 cell line, whereas the highest $\mathrm{IC}_{50}$ was observed against $\mathrm{RD}$ cell line. $\mathrm{IC}_{50}$ for $\mathrm{L} 20 \mathrm{~B}$ cell line was $448 \mu \mathrm{g} / \mathrm{mL}$ (Fig. A4 in Additional file 5). In case of RD, complete
Table 4 Antimicrobial potential of the most active phytoconstituents of Symplocos racemosa bark against drugresistant strains and some clinical isolates of MRSA

\begin{tabular}{|c|c|c|c|}
\hline & \multirow[t]{2}{*}{ Microorganisms } & \multicolumn{2}{|c|}{ Average zone of inhibition $(\mathrm{mm})^{*}$} \\
\hline & & Flavonoids & Cardiac glycosides \\
\hline & CRIRS 1 & $15.33 \pm 0.881$ & - \\
\hline & CRIRS 2 & $15.66 \pm 0.333$ & - \\
\hline & CRIRS 3 & $16 \pm 0.577$ & - \\
\hline & CRIRS 4 & $17.33 \pm 0.333^{\mathrm{a}}$ & $14.66 \pm 0.333^{b}$ \\
\hline & CRIRS 5 & $17 \pm 0.577^{\mathrm{a}}$ & $12.33 \pm 0.333^{b}$ \\
\hline & CRIRS 6 & $16.66 \pm 0.333^{a}$ & $13 \pm 0^{b}$ \\
\hline & CRIRS 7 & $22.66 \pm 0.666$ & - \\
\hline \multirow[t]{19}{*}{ Group 1} & CRIRS 8 & $17.66 \pm 0.333^{a}$ & $13.66 \pm 0.333^{b}$ \\
\hline & CRIRS 9 & $14.66 \pm 0.881$ & - \\
\hline & CRIRS 10 & $15.33 \pm 0.881^{a}$ & $14.33 \pm 0.333^{\mathrm{a}}$ \\
\hline & CRIRS 11 & $15 \pm 0.577$ & - \\
\hline & CRIRS 12 & $15.66 \pm 0.666$ & - \\
\hline & CRIRS 13 & $19.66 \pm 0.333^{a}$ & $14 \pm 0^{b}$ \\
\hline & CRIRS 14 & $18.66 \pm 0.333$ & - \\
\hline & CRIRS 15 & $14.66 \pm 0.333$ & - \\
\hline & CRIRS 16 & $16.33 \pm 0.881$ & - \\
\hline & CRIRS 17 & $20 \pm 0$ & - \\
\hline & CRIRS 18 & $19.33 \pm 0.333$ & - \\
\hline & CRIRS 19 & $16 \pm 0$ & - \\
\hline & CRIRS 20 & $15.33 \pm 0.333$ & - \\
\hline & CRIRS 21 & $17.66 \pm 0.333$ & - \\
\hline & CRIRS 22 & $18.66 \pm 0.333$ & - \\
\hline & DSECI 01 & $21.33 \pm 0.333^{a}$ & $14 \pm 0^{\mathrm{b}}$ \\
\hline & DSECI 02 & $20 \pm 0^{\mathrm{a}}$ & $17.33 \pm 0.666^{b}$ \\
\hline & DSECI 03 & $27.66 \pm 0.333^{a}$ & $16.66 \pm 0.881^{b}$ \\
\hline & DSECI 04 & $28.33 \pm 0.333^{a}$ & $18 \pm 0^{b}$ \\
\hline \multirow[t]{8}{*}{ Group 2} & DSECI 05 & $24.33 \pm 0.666^{a}$ & $15 \pm 0^{b}$ \\
\hline & DSECI 06 & $22.33 \pm 0.333^{a}$ & $14.33 \pm 0.333^{b}$ \\
\hline & DSECI 07 & $26.33 \pm 0.666$ & - \\
\hline & DSECI 08 & $22 \pm 0.577^{a}$ & $14.33 \pm 0.666^{b}$ \\
\hline & DSECI 09 & $25.66 \pm 0.333$ & - \\
\hline & DSECI 10 & $27.33 \pm 0.333$ & - \\
\hline & DSECI 11 & - & - \\
\hline & DSECI 12 & $23.33 \pm 0.333^{a}$ & $15.66 \pm 0.333^{b}$ \\
\hline
\end{tabular}

Similar alphabetic letters $(a, a)$ on the values within a row represent no significant difference at $95 \%$ confidence level, as indicated by One-way ANOVA followed by Tukey's t-test

*Values are expressed as Mean \pm Standard error of means (SEM) $(n=3)$

rounding off of the cells was seen upto the concentration of $2.5 \mathrm{mg} / \mathrm{mL}$, and thereafter the inhibitory effect gradually reduced down the concentration gradient. For Hep2 and L20B, rounding off of the cells could be seen upto $1.25 \mu \mathrm{g} / \mathrm{mL}$ and $0.625 \mu \mathrm{g} / \mathrm{mL}$, respectively. The 
flavonoids showed a negligible effect on the normal cell line, i.e., Vero cell line (only 9.56-15.24\%), in comparison to the \% inhibition obtained against the three cancerous cell lines at the various tested concentrations (Fig. A4 in Additional file 5), thus exhibiting a very high $\mathrm{IC}_{50}$ value $(>10,000 \mu \mathrm{g} / \mathrm{mL})$, in comparison to that obtained against cancerous cell lines $(361-494 \mu \mathrm{g} / \mathrm{mL})$.

\section{In-vivo acute oral toxicity studies}

The flavonoids, further tested for their toxicity in mice, did not show any toxic effects during the whole experimentation period as evident by the normal behavior and sleep patterns, salivation, breathing rate as well as the absence of any abnormalities on the skin, fur, eyes etc. No significant difference was seen between the weights of the tested (6/6) and the control (untreated) group (6/ 6) (Fig.A5 in Additional file 5). The weight of the vital organs obtained from each test group (6/6) showed no statistical difference from the control group (Table 5). The kidney and liver functioning remained normal in the test group (6/6) was completely normal since no statistical difference was seen between the various kidney and liver-functioning parameters of the treated and control groups of both male and female mice (Table 6). Further, the general and histopathological evaluation showed normal color, texture, architectural details and structural organization of the three organs under study (Fig. 2).

\section{Detection of the probable bioactive components in Symplocos racemosa flavonoids}

The bioactive compounds, which may be responsible for the antimicrobial efficacy of Symplocos racemosa flavonoids, were detected by separation using PreparativeThin Layer Chromatography followed by their antimicrobial screening against two Gram positive, two Gram negative and one yeast strain. The most active band was then subjected to Gas Chromatography-Mass Spectrometry (GC-MS) to identify the antimicrobial components present in it.

\section{Thin layer chromatography (TLC) analysis}

The flavonoids were best resolved using the solvent system consisting of Butanol, Ethanol and water in the ratio 4:1:2. This solvent system separated the flavonoids into 4 bands having a Retention Factor $\left(R_{\mathrm{f}}\right)$ in the range of 0.151-0.769. When observed under natural light, only 2 Bands were visible. Band 1 was seen as a dark brown spot while Band 2 was a light brown spot (Fig. 3a). All the four bands were visible when observed in iodine vapors and UV short wavelength $(264 \mathrm{~nm})$. Upon staining with iodine vapors, Band 1 was seen as the intensely brown spot and the other three bands showed lightbrown intensity (Fig. 3b), whereas these bands could be seen as blue spots under UV short wavelength $(264 \mathrm{~nm})$. Here, Band 1 was intense in comparison to the other three bands (Fig. 3c). When observed under long wavelength UV at $365 \mathrm{~nm}$, only Band 1 could be seen as a dark blue spot against the blue background while the other bands were not visible (Fig. 3d).

\section{Quantitative separation of the bands using preparative- TLC (P-TLC) method and their antimicrobial screening} In case of Symplocos racemosa, $76.66 \mathrm{mg}$ dry wt. of the flavonoids, when subjected to P-TLC, resulted in the separation of 4 bands (S1-S4) which were obtained with a dry wt. of: S1 (5 mg), S2 (8 mg), S3 (12 mg), S4 (9 mg). These were dissolved in methanol and a $20 \mu \mathrm{L}$ aliquot was tested for their antimicrobial potential. Band S3 was significantly active against all the five test organisms. Band S1 was weakly effective against Klebsiella pneumoniae 1 and Candida albicans but was quite active against Staphylococcus epidermidis. Band S2 was negligibly active against Staphylococcus aureus and Candida

Table 5 Absolute and relative organ weight of control and treated mice (male and female) in the acute toxicity study of Symplocos racemosa flavonoids

\begin{tabular}{|c|c|c|c|c|c|c|}
\hline \multirow[b]{2}{*}{ Organs } & \multicolumn{2}{|c|}{ Absolute organ weight $(g)^{a}$} & \multirow[b]{2}{*}{$p$-value } & \multicolumn{2}{|c|}{ Relative organ weight $(\%)^{a}$} & \multirow[b]{2}{*}{$p$-value } \\
\hline & Control & Treated & & Control & Treated & \\
\hline & & & MALE & & & \\
\hline Liver & $0.724 \pm 0.031$ & $0.692 \pm 0.013$ & 0.365 & $2.453 \pm 0.031$ & $2.441 \pm 0.007$ & 0.715 \\
\hline Kidneys & $0.401 \pm 0.022$ & $0.379 \pm 0.015$ & 0.437 & $1.355 \pm 0.035$ & $1.334 \pm 0.026$ & 0.638 \\
\hline \multirow[t]{2}{*}{ Heart } & $0.165 \pm 0.007$ & $0.157 \pm 0.005$ & 0.390 & $0.558 \pm 0.008$ & $0.577 \pm 0.008$ & 0.137 \\
\hline & & & FEMALE & & & \\
\hline Liver & $0.827 \pm 0.044$ & $0.783 \pm 0.029$ & 0.435 & $2.613 \pm 0.055$ & $2.653 \pm 0.060$ & 0.638 \\
\hline Kidneys & $0.410 \pm 0.023$ & $0.368 \pm 0.006$ & 0.131 & $1.297 \pm 0.031$ & $1.248 \pm 0.006$ & 0.179 \\
\hline Heart & $0.166 \pm 0.0118$ & $0.150 \pm 0.004$ & 0.254 & $0.524 \pm 0.0195$ & $0.509 \pm 0.009$ & 0.651 \\
\hline
\end{tabular}

${ }^{2}$ Data indicate Mean \pm SEM ( $n=6$ for each group). There was no significant difference between the test and control groups as indicated by Post hoc Tukey's t-test 
Table 6 Effect of Symplocos racemosa flavonoids on biochemical parameters in acute oral toxicity study

\begin{tabular}{|c|c|c|c|c|c|c|}
\hline \multirow[t]{2}{*}{ Parameters } & \multicolumn{2}{|l|}{ Male } & \multirow[b]{2}{*}{$p$-value } & \multicolumn{2}{|l|}{ Female } & \multirow[b]{2}{*}{$p$-value } \\
\hline & Control $^{a}$ & Treated $^{\mathrm{a}}$ & & Control $^{a}$ & Treated $^{\mathrm{a}}$ & \\
\hline Urea $(\mathrm{Mmol} / \mathrm{L})$ & $42.633 \pm 0.839$ & $42.460 \pm 0.553$ & 0.867 & $40.13 \pm 0.991$ & $40.183 \pm 0.539$ & 0.963 \\
\hline Creatinine ( $\mu \mathrm{mol} / \mathrm{L})$ & $0.774 \pm 0.026$ & $0.765 \pm 0.019$ & 0.799 & $0.618 \pm 0.024$ & $0.601 \pm 0.012$ & 0.535 \\
\hline Total bilirubin $(\mu \mathrm{mol} / \mathrm{L})$ & $2.376 \pm 0.093$ & $2.371 \pm 0.063$ & 0.882 & $1.76 \pm 0.025$ & $1.772 \pm 0.014$ & 0.677 \\
\hline Aspartate aminotransferase (AST)(U/L) & $208.96 \pm 4.192$ & $209.11 \pm 2.312$ & 0.976 & $160.66 \pm 2.708$ & $159.76 \pm 1.574$ & 0.780 \\
\hline Alanine aminotransferase (ALT) $(\mathrm{U} / \mathrm{L})$ & $79.95 \pm 2.095$ & $81.698 \pm 0.591$ & 0.441 & $61.33 \pm 2.677$ & $61.501 \pm 1.338$ & 0.955 \\
\hline Alkaline phosphatase (ALP) (U/L) & $258.3 \pm 2.510$ & $256.89 \pm 1.344$ & 0.633 & $183.33 \pm 1.612$ & $181.733 \pm 0.794$ & 0.403 \\
\hline
\end{tabular}

${ }^{a}$ Values are expressed as mean \pm SEM ( $n=6$ for each group). There was no significant difference $(p>0.05)$ between the test and control groups as indicated by one way ANOVA followed by Post hoc Tukey's t-test

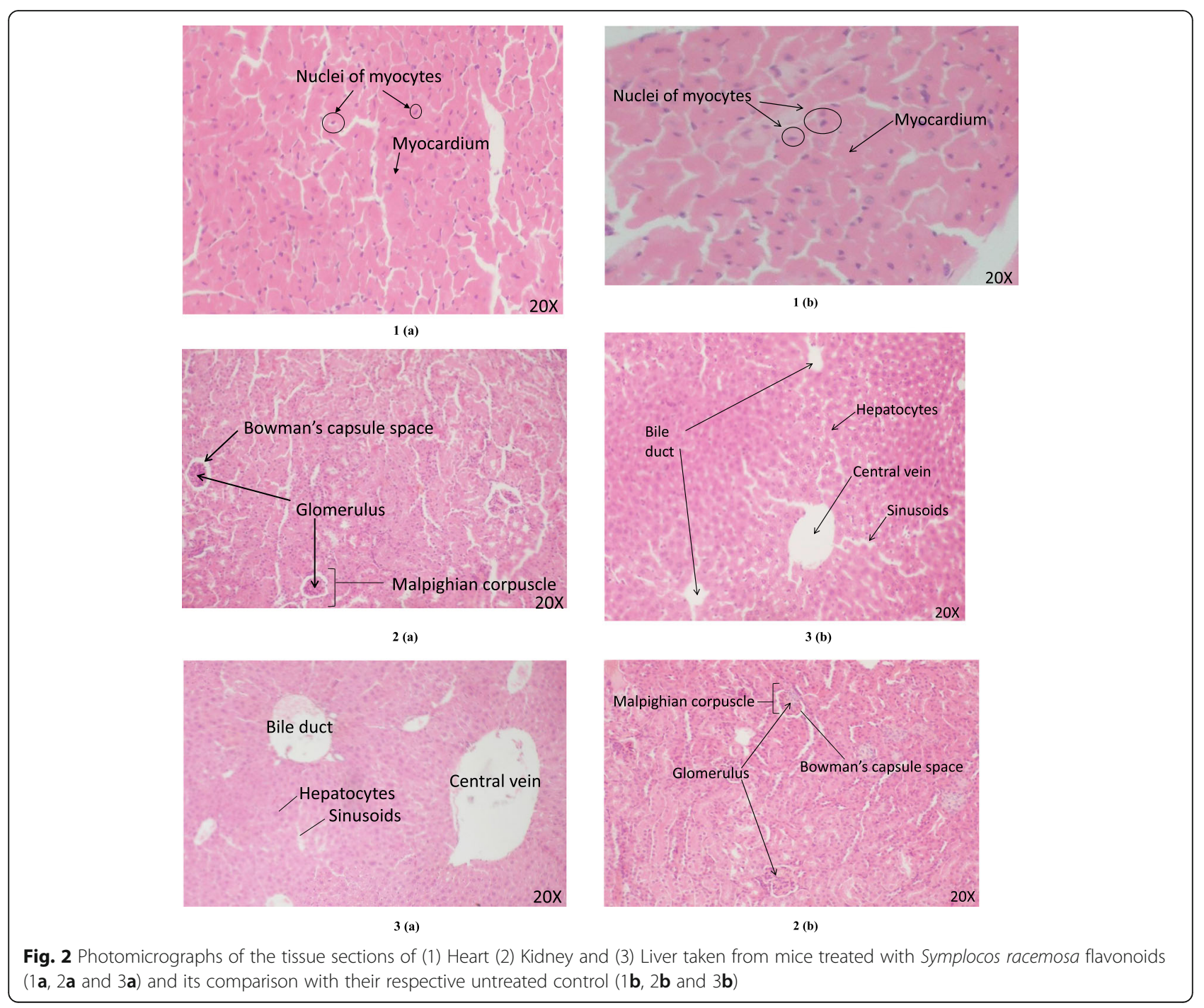




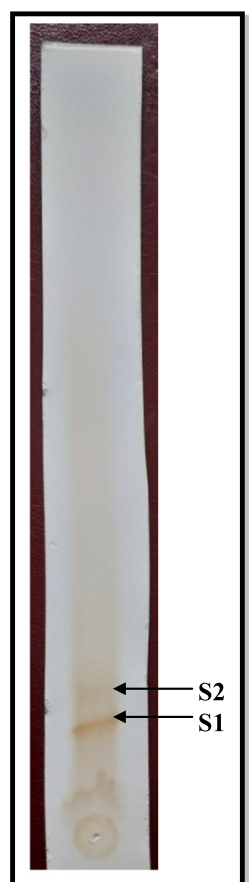

(a)

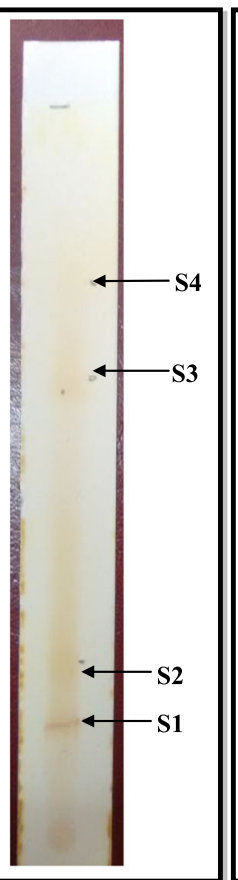

(b)

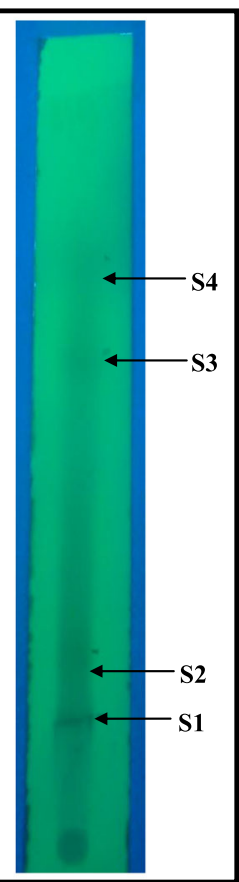

(c)

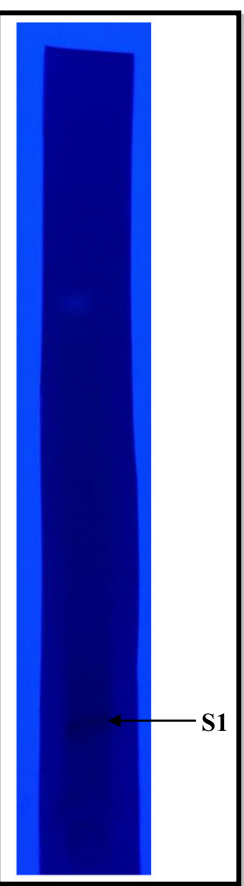

(d)

Fig. 3 TLC chromatograms of the Symplocos racemosa flavonoids revealing the presence of various bands under a Natural light $\mathbf{b}$ Stained with lodine vapors c UV light (264 nm) d UV light (365 nm)

albicans, but did not show any activity against Klebsiella pneumoniae 1, Staphylococcus epidermidis and Shigella flexneri. Band S4 was only active against Staphylococcus epidermidis. Since, Band S3 was significantly active against the test organisms; it was taken up further for spectroscopic studies.

Identification of the compounds present in the active band 3 (S3) by Gas Chromatography-Mass Spectrometry (GC-MS) analysis

Symplocos racemosa flavonoids, when subjected to PTLC analysis revealed the presence of 4 bands, out of which Band 3 (S3) was found to be antimicrobially most active, when screened against various test organisms. Its GC-MS analysis revealed the presence of mixture of a number of compounds at RTs falling within the range of 3.22-34.40 (Fig. 4; Additional file 6). The library search led to detection of a number of compounds at each RT (Table 7). Based on the abundance in terms of area \%, compounds at three RTs were found to be major compounds in this active band, which were identified as Bicyclo [2.2.1] heptan-2-one,1,7,7-trimethyl-, (1S)- at an RT of 11.14; Silane, Dimethyl(dimethyl(dimethyl(2-isopropylphenoxy)silyloxy)silyloxy)(2-isopropylphenoxy)- at an RT of 13.47 and 7,11b-Dihydro-6H-indeno[2,1-c] chromene-3,4,6a,9,10-pentol pentakis (trimethylsilyl)ether at an RT of 34.40 .

\section{Discussion}

Antibiotics have always been bliss for the human civilization; however, their irrational and irresponsible use has significantly contributed to antimicrobial resistance. There is a promising future for the medicinal plants' usage and their hidden potential could be decisive in changing the course of medical history [37]. Keeping this in mind, the present study aimed at scientifically exploring the bioactive potential of Symplocos racemosa. It has been observed that most of the antimicrobial compounds identified in plants are mostly aromatic or saturated organic molecules, which can easily be solubilized in organic solvents [38] and in the present study, ethyl acetate was able to extract out the antimicrobial components from Symplocos racemosa to a maximum extent, followed by butanol and hexane. The better antimicrobial potential of the ethyl acetate extract may be attributed to the better solubility of such residues in ethyl acetate [39]. The presence of major groups like flavonoids, cardiac glycosides, saponins, tannins, triterpenes and phytosterols are in consonance with other reports on this plant $[40,41]$ and other medicinal plants $[17,42]$. Cardiac glycosides were the most abundant followed by flavonoids whereas triterpenes were present in least quantity. Quite encouragingly, flavonoids and cardiac glycosides were effective against 12 and 9 strains respectively and our results are well supported by the previous observations for various plant parts of Moringa 


\section{CIL/ SAIF Panjab University Chandigarh}

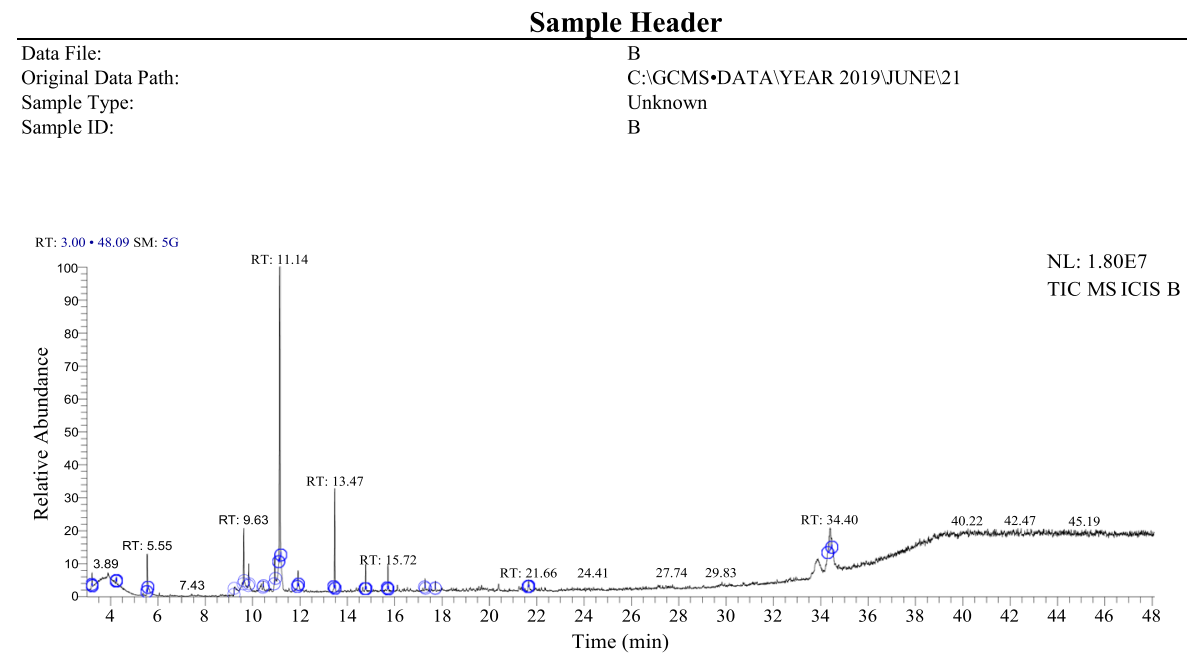

Qual Peak Table

\begin{tabular}{rrrr}
\hline RT & Peak Area & Area $\%$ & Peak Height \\
\cline { 4 - 4 } 4.22 & 721881.07 & 1.14 & 693994.73 \\
5.25 & 160089.03 & 0.25 & 230579.60 \\
5.55 & 2907723.75 & 4.59 & 2019768.89 \\
9.22 & 141733.43 & 0.22 & 240523.84 \\
9.63 & 4524195.64 & 7.14 & 2949956.82 \\
9.84 & 1478517.41 & 2.33 & 1151840.21 \\
10.46 & 321694.31 & 0.51 & 316816.30 \\
10.94 & 2564603.95 & 4.05 & 1537426.31 \\
11.14 & 28588424.25 & 45.13 & 15951170.33 \\
11.93 & 1291139.96 & 2.04 & 794475.39 \\
13.47 & 8128156.37 & 12.83 & 5408657.63 \\
14.78 & 1931709.86 & 3.05 & 1328515.79 \\
15.72 & 1837936.22 & 2.90 & 1351520.63 \\
17.29 & 529468.59 & 0.84 & 487257.30 \\
17.72 & 397361.77 & 0.63 & 391171.61 \\
21.66 & 546416.71 & 0.86 & 376776.36 \\
34.40 & 7279061.62 & 11.49 & 1201517.65
\end{tabular}

Fig. 4 The GC-MS Chromatogram of the active band S3 obtained from the flavonoids of Symplocos racemosa bark

oleifera $[43,44]$, where these were quite effective against the Gram positive, Gram negative and the yeast strains. The significance of the study can be gauged from the fact that these phytoconstituents were highly effective against resistant microbes e.g., MRSA and Candida albicans, which cause severe infections in hospitals/healthcare settings, which is in line with another study on Eugenia jambolana seed extracts which was effective against MDR human pathogens [45]. The antimicrobial potential of phytoconstituents is well-supported by the fact that these substances serve as plant defense mechanisms against predation by microorganisms and that the wide array of such microbial infections could have triggered the synthesis of such vast variety of phytoconstituents with a wide and variable spectrum [38]. The results obtained from ADA and MIC is supportive of each other. MIC determination is an effective method for evaluating the efficacy of an antimicrobial [46], where all the extracts were quite effective against MRSA, Candida albicans, Pseudomonas aeruginosa and Escherichia coli etc., which highlights the importance of the study. The MIC values obtained in this study $(0.5-10 \mathrm{mg} / \mathrm{mL})$ were comparable to or lower than the values obtained for other medicinal plants' extracts, viz., $0.4-4 \mathrm{mg} / \mathrm{mL}$ [22]; $2.5-5 \mathrm{mg} / \mathrm{mL}$ [47], $5-20 \mathrm{mg} / \mathrm{mL}$ [48]. VCC studies give an assessment of the microbistatic/cidal effect of the compound, which further endorsed the potential of this medicinal plant, as it showed a significant bactericidal effect against MRSA, Escherichia coli, Pseudomonas aeruginosa, Staphylococcus aureus, Staphylococcus epidermidis etc. The PAE gives an idea of the time period upto which the compound is effective post-exposure and hence could be a decisive factor for development of any natural compound into a useful drug. The PAE in this study ranged from 2 to $6 \mathrm{~h}$. The in vitro biosafety evaluation of the ethyl acetate extract and phytoconstituents by Ames and MTT assay proved them to be nonmutagenic and non-cytotoxic, respectively. 
Table 7 The compounds detected in the active band S3 obtained from the flavonoids of Symplocos racemosa bark using GC-MS

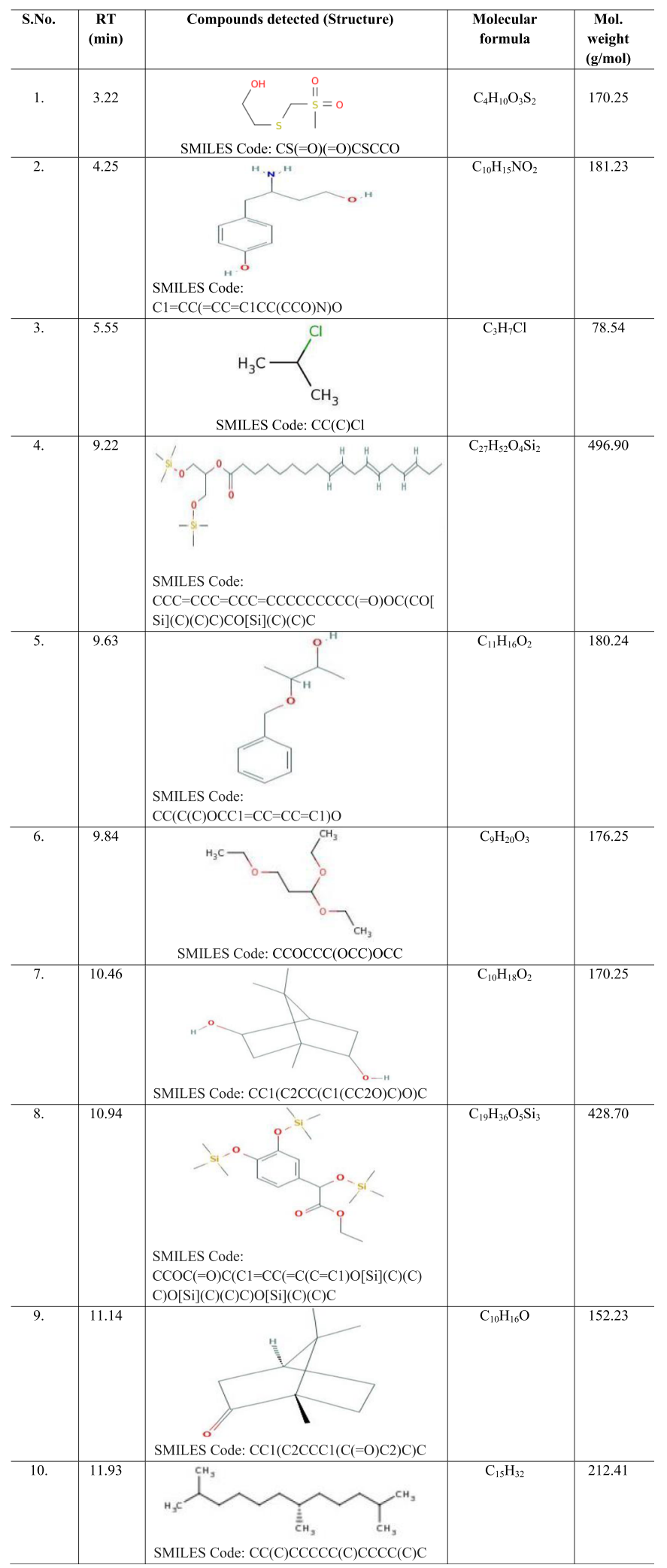


Table 7 The compounds detected in the active band S3 obtained from the flavonoids of Symplocos racemosa bark using GC-MS (Continued)

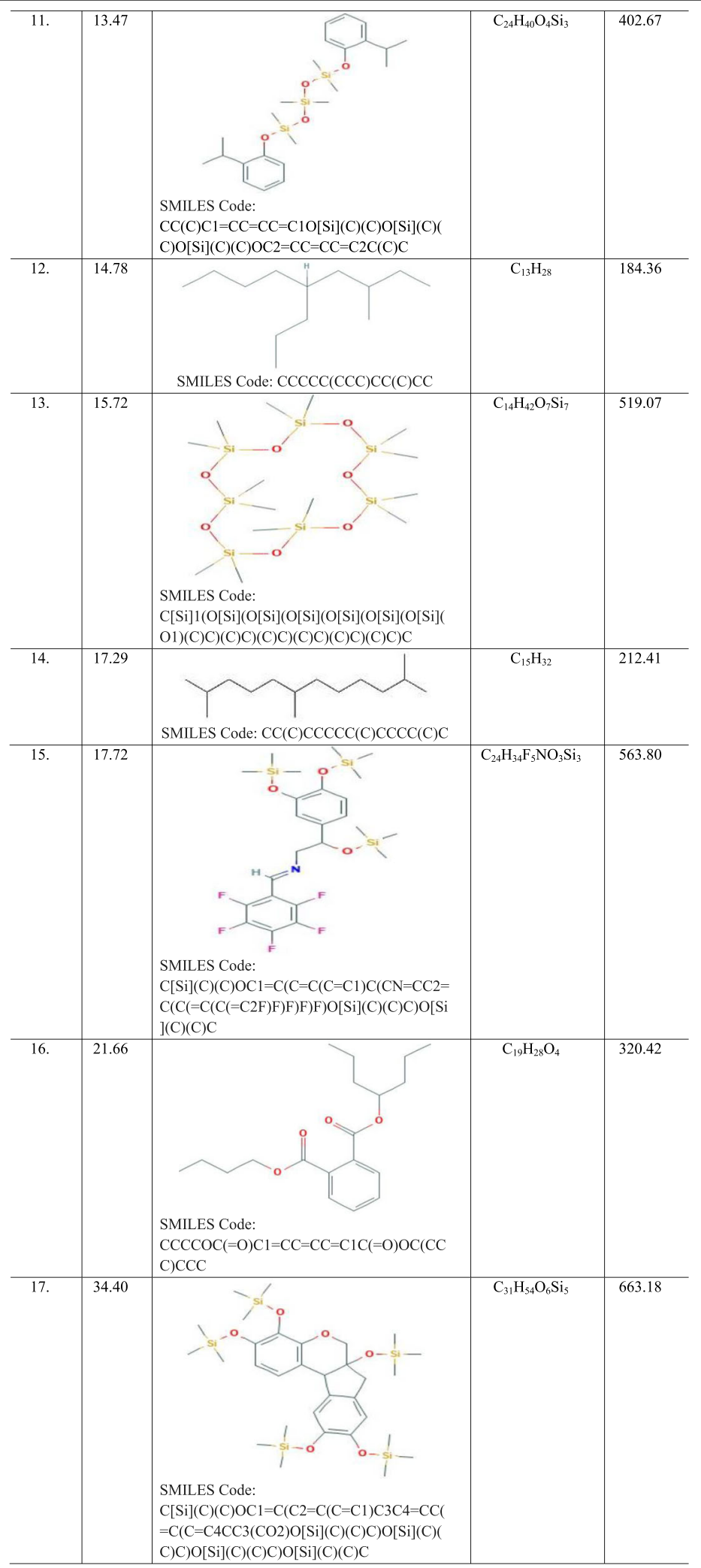


It is a well-known fact that the biofilms-formers are a leading cause of the rising cases of the chronic infectious diseases and recurrent infections. Therefore, bioactive compounds which are able to modulate/ disrupt the biofilm forming ability, have an exceptional importance in the field of drug development [49]. The promising antibiofilm potential of flavonoids and cardiac glycosides highlight the importance of the study, where the former were more effective [50]. This could be justified by the fact that the plant metabolites such as flavonoids, phenolic acids etc. reportedly possess antibiofilm activity [51, 52]. The potency was also tested against some drugresistant strains of E.coli, Salmonella spp. and MRSA, where flavonoids and cardiac glycosides showed a broad spectrum activity, thus, justifying its candidature as a potential antimicrobial and antibiofilm drug of future. To add up on the bioactive potential of the plant, the most active phytoconstituents, i.e., flavonoids were screened for their cytotoxicity against three cancerous cell lines and our results are in consonance with those obtained for compounds of Acanthus hirsutus Boiss (11.17$700 \mu \mathrm{g} / \mathrm{mL})$, Euphorbia hirta $(625 \mu \mathrm{g} / \mathrm{mL})[53,54]$ and some naturally occurring quinones [55] .

To further strengthen the biosafety aspect, acute oral toxicity of its flavonoids was evaluated in mice. Toxicological evaluation is an important aspect of pharmacology for assessing any negative effect of the bioactive substance on living organisms prior to its clinical usage by humans. This aspect is crucially important as toxicity results from animals, especially mice and rats, help in judging the safety of the compounds, since mice and rats have an anatomy close to that of human body and any effect on the animal model would clearly indicate towards its possible toxic effects. Abundant literature is available validating the ethno-medical knowledge on the significance of medicinal plants in prevention and treatment of various diseases. However, the medicinal plants contain a complex mixture of many bioactive phytochemical groups with diverse mode of action, which may possibly show any adverse effects upon interaction with the human/animal cells. Thus, it is of utmost importance to investigate the safety and biological properties of the medicinal plants prior to their usage. The biosafety of the flavonoids was thus confirmed since no signs of abnormality, illness or biochemical and pathological changes were observed in the test group of animals, indicating a normal metabolism and growth. These results are in line with other allied studies reporting the nontoxicity of the medicinal plants/ extracts [12, 16-18].

So as to establish the active compounds which impart a significant and broad spectrum antimicrobial potential to the most active groups of phytoconstituents (flavonoids), the chromatographic and spectroscopic techniques such as TLC and GC-MS methods were employed. The chromatogram revealed the presence of several bands (S1S4) with varying $R_{f}$ values. The number of separated bands, corresponding to the number of separated compounds, strictly depended on the solvent system used. It means that the separation of bands depends on the nature and interaction of the compounds being separated with the solvents of the used system. The bands obtained were quantitatively separated using preparative-TLC method, where one band i.e., S3 was found to be the active with a broad spectrum. Hence, it was further subjected to GC-MS analysis so as to identify the antimicrobial components in this active band. Interestingly, the presence of compound Bicyclo[2.2.1]heptan-2-one,1,7,7-trimethyl-, (1S)- as one of the major compounds in band S3 corresponded well with a report on the GC-MS analysis of the methanolic extract of Coriandrum sativum leaves, where this compound was identified as one of the major bioactive phytochemical compounds and possessed antimicrobial activity, which provided further credence to the study [56]. Its presence as a major phytochemical compound was also in concordance with other studies on chloroformic extract of Acacia karoo root and Artemisia lavandulaefolia essential oil, where Bicyclo[2.2.1]heptan-2-one,1,7,7-trimethyl-, (1S)- was present as a major bioactive phytochemical constituent $[57,58]$. Also, another major compound "Silane, Dimethyl(dimethyl(dimethyl(2-isopropylphenoxy)silyloxy)silyloxy)(2-isopropylphenoxy)-" was also identified as a metabolic component of a wild edible mushroom Pleurotus cornucopiae (Paulet), by GC/MS [59]. Similarly, this compound has also been reported as a phyto-compound present in the extract of Terminalia arjuna upon its GC-MS analysis [60]. Similarly, compound "7,11b-Dihydro-6H-indeno[2,1-c] chromene 3, 4,6a,9,10-pentol pentakis (trimethylsilyl)ether" has also been reported as a major bioactive components in the GC-MS analysis of the ethanol extract from leaves of Combretum albidum [61].

\section{Conclusion}

The present study thus provides a scientific support to the use of Symplocos racemosa bark to explore its bioactive compounds of pharmaceutical importance, as evident from their wide zone of inhibition, low MIC values, shorter killing time and extended post antibiotic effect. These compounds showed a significant antibiofilm potential, were potent against drug resistant strains and were cytotoxic against the cancerous cell lines, which further add credence to the bioactive potential of the plant. The ethyl acetate extract and the phytoconstituents had a biosafe profile as seen in Ames test, MTT assay and acute oral toxicity studies. The various bioactivities exhibited by Symplocos racemosa revealed and strengthened its candidature for development of potent pharmaceutical drugs. 


\section{Supplementary Information}

The online version contains supplementary material available at https://doi. org/10.1186/s40360-020-00453-y.

Additional file 1:. Organic extracts' preparation and their antimicrobial screening; Minimum Inhibitory Concentration (MIC); Qualitative and Quantitative analysis for the detection of major group of phytoconstituents; Ames Mutagenicity Test and MTT assay Protocol.

Additional file 2: Detailed protocol for Antibiofilm potential of the phytoconstituents of Symplocos racemosa bark; and Antimicrobial potential of the phytoconstituents against drug- resistant clinical isolates of MRSA, Escherichia coli, Enterococcus sp. and Salmonella spp.

Additional file 3:. In vitro cytotoxicity studies by MTT assay against RD, L2OB and Hep2 cell lines.

Additional file 4: Acute Oral Toxicity study of Symplocos racemosa flavonoids in Swiss albino mice.

Additional file 5: Figures A1-A6 and Table TI, T2 and $\mathbf{T 3}$ of the main manuscript.

Additional file 6: GC mass report of the compounds detected in band S3 of flavonoids from Symplocos racemosa bark.

\section{Abbreviations}

ADA: Agar Diffusion Assay; CPCSEA: Committee for the Purpose of Control and Supervision of Experiments on Animals; IAEC: Institutional Animal Ethics Committee; MIC: Minimum Inhibitory Concentration; MRSA: MethicillinResistant Staphylococcus aureus; MTCC: Microbial Type Culture Collection; MTT: [3-(4,5-dimethylthiazol-2-yl)-2,5-diphenyl tetrazolium bromide]; PAE: Post Antibiotic Effect; TAP: Total Activity Potency

\section{Acknowledgements}

The help extended by Mr. Yogesh Kumar Sood (Deputy Assistant Director) and his staff at Central Research Institute (C.R.I), Kasauli (H.P.), India, is duly acknowledged. The help provided for GC-MS analysis by Sophisticated AnaIytical Instrumentation Facility (SAIF), Panjab University, Chandigarh is also highly acknowledged.

\section{Authors' contributions}

DSA: As a Principle Investigator (PI), guided through the experimental design and data analysis. HS: Performed the experimental work and drafted the manuscript. YK: Guided in performing the the in vivo experimentation and in vitro cytotoxic activity against cell lines. VKG: Carried out the histopathological examination and its interpretation. All authors have read and approved the manuscript.

\section{Funding}

The work has been supported by financial assistance provided by Guru Nanak Dev University, Amritsar to Dr. Daljit Singh Arora under UGC-UPE scheme granted by University Grants Commission, New Delhi (India). Henna Sood is thankful to University Grants Commission for UPE fellowship vide letter no. [8015/Estt./A-2 dated 16/04/2013].

\section{Availability of data and materials}

The raw datasets generated and/or analyzed during the current study are not publicly available [being a part of the Ph.D. thesis of the first author] but are available from the corresponding author on reasonable request.

\section{Ethics approval and consent to participate}

All procedures performed in studies involving animals were in accordance with the ethical standards of the institution or practice at which the studies were conducted. Ethics approval has been provided by the Institutional Animal Ethics Committee (IAEC) of Central Research Institute (C.R.I), Kasauli (H.P.), India, working under the guidance of Committee for the Purpose of Control and Supervision of Experiments on Animals (CPCSEA), New Delhi, India vide no. CPCSEA/IAEC/CRI/14-114-2016. The written informed consent to use the animals in this study was obtained from the Animal house of Central Research Institute, Kasauli (H.P.).

\section{Consent for publication}

Not applicable.

\section{Competing interests}

The authors declare that they have no conflict of interest, i.e., the submitted work was not carried out in the presence of any personal, professional or financial relationships.

\section{Author details}

${ }^{1}$ Microbial Technology Laboratory, Department of Microbiology, Guru Nanak Dev University, Amritsar 143005, India. ${ }^{2}$ National Salmonella \& Escherichia Centre and Diagnostic Reagents Laboratory, Central Research Institute, Kasauli, HP 173204, India. ${ }^{3}$ Department of Veterinary Pathology, Dr. G.C.Negi College of Veterinary and Animal Sciences, CSK Himachal Pradesh KrishiVishvavidyalaya, Palampur, HP 176062, India.

Received: 18 March 2020 Accepted: 21 October 2020 Published online: 17 November 2020

\section{References}

1. Richardson LA. Understanding and overcoming antibiotic resistance. PLoS Biol. 2017. https://doi.org/10.1371/journal.pbio.2003775

2. Menghani E, Pareek A, Negi RS, Ojha CK. Search for antimicrobial potentials from certain Indian medicinal plants. Res J Med Plants. 2011;5:295-301.

3. Khan A, Ahmad A, Akhtar F, Yousuf S, Xess I, Khan LA, et al. Ocimum sanctum essential oil and its active principles exert their antifungal activity by disrupting ergosterol biosynthesis and membrane integrity. Res Microbiol. 2010;161:816-23.

4. Endo EH, Cortez DAG, Ueda-Nakamura T, Nakamura CV, Dias Filho BP. Potent antifungal activity of extracts and pure compound isolated from pomegranate peels and synergism with fluconazole against Candida albicans. Res Microbiol. 2010:161:534-40.

5. Saritha K, Rajesh A, Manjulatha K, Setty OH, Yenugu S. Mechanism of antibacterial action of the alcoholic extracts of Hemides musindicus (L.) R. Br. ex Schult, Leucasa spera (Wild.), Plumbago zeylanica L., and Tridax procumbens (L.) R. Br. ex Schult. Front Microbiol. 2015:6:577.

6. Silva AP, Nascimento da Silva LC, da Fonseca M, Caíque S, de Araújo JM, Correia MT, MDS C, Lima VL. Antimicrobial Activity and Phytochemical Analysis of Organic Extracts from Cleome spinosa Jaqc. Front Microbiol. 2016;7:963.

7. Hernandez NE, Tereschuk ML, Abdala LR. Antimicrobial activity of flavonoids in medicinal plants from Tafidel Valle (Tucuman, Argentina). J Ethnopharmacol. 2000;73:317-22.

8. Orhan DD, Özçelik B, Özgen S, Ergun F. Antibacterial, antifungal, and antiviral activities of some flavonoids. Microbiol Res. 2010;165:496-504.

9. Achika JI, Arthur DE, Gerald I, Adedayo A. A review on the phytoconstituents and related medicinal properties of plants in the Asteraceae family. IOSR J Appl Chem. 2014;7:1-8.

10. Irfan M, Ahmed S, Sharma M. Antimicrobial activity of terpenoids from Sphaeranthusindicus. L Asian J Plant Sci Res. 2014;4:1-6.

11. Tagousop CN, Ekom SE, Ngnokam D, Voutquenne-Nazabadioko L. Antimicrobial activities of flavonoid glycosides from Graptophyllum grandulosum and their mechanism of antibacterial action. BMC Complement Alternat Med. 2018;18:252.

12. Ping KY, Darah I, Chen Y, Sreeramanan S, Sasidharan S. Acute and subchronic toxicity study of Euphorbia hirta L. methanol extract in rats. BioMed Res Int. 2013. https://doi.org/10.1155/2013/182064.

13. Jadhav M, Menon SK, Shailajan S. Anti-androgenic effect of Symplocos racemosa Roxb.against letrozole induced polycystic ovary using rat model. J Coast Life Med. 2013;1:309-14.

14. Bhusnar HU, Nagore DH, Nipanikar SU. Phytopharmacological profile of Symplocos racemosa: a review. Pharmacologia. 2014;5:76-83.

15. Sood H, Kaur H, Arora DS. Statistical optimization of physiochemical parameters for enhancing the antimicrobial potential of Lodhra (Symplocos racemosa) bark and its biosafety evaluation. Int J Pharm. 2015;5:852-66.

16. Jothy SL, Zakaria Z, Chen Y, Lau YL, Latha LY, Sasidharan S. Acute oral toxicity of methanolic seed extract of Cassia fistula in mice. Molecules. 2011 16:5268-82.

17. Mahajan H, Arora DS, Singh H, Jain SK, Namarta K, Singh J. Scientific validation of the antimicrobial and antiproliferative potential of 
Clerodendrum serratum (L.) moon, its phytoconstituents and their biosafety by acute oral toxicity study. Drug Chem Toxicol. 2019;38:1-11.

18. Murbach TS, Hirka G, Szakonyine JP, Gericke N, Endres JR. A toxicological safety assessment of a standardized extract of Sceletium tortuosum (Zembrin) in rats. Food Chem Toxicol. 2014;74:190-9.

19. Kaur GJ, Arora DS. Antibacterial and phytochemical screening of Anethum graveolens, Foeniculum vulgare and Trachyspermum ammi. BMC Complement Altern Med. 2009;9:30.

20. Ezeonu CS, Ejikeme CM. Qualitative and quantitative determination of phytochemical contents of indigenous Nigerian softwoods. New J Sci. 2016. https://doi.org/10.1155/2016/5601327.

21. Arora DS, Sood H. In vitro antimicrobial potential of extracts and phytoconstituents from Gymnema sylvestre R.Br. leaves and their biosafety evaluation. AMB Expr. 2017;7:115

22. Arora DS, Onsare JG. In vitro antimicrobial evaluation and phytoconstituents of Moringa oleifera pod husks. Ind Crop Prod. 2014a;52:125-35.

23. Raja AF, Ali F, Khan IA, Shawl AS, Arora DS, Shah BA, Taneja SC. Antistaphylococcal and biofilm inhibitory activities of acetyl-11-keto- $\beta$ boswellic acid from Boswellia serrata. BMC Microbiol. 2011;11:54.

24. Eren Y, Ozata A. Determination of mutagenic and cytotoxic effects of Limonium globuliferum aqueous extracts by Allium, Ames and MTT tests. Rev Bras Farmacogn. 2014;24:51-9.

25. Stepanovic S, Vukovi D, Hola V, Di Bonaventura G, Djukić S, Cirkovic I, Ruzicka F. Quantification of biofilm in microtiter plates: overview of testing conditions and practical recommendations for assessment of biofilm production by Staphylococci. APMIS. 2007;115:891-9.

26. Costa GA, Rossatto FC, Medeiros AW, Correa APF, Brandelli A, Frazzon APG Motta ADS. Evaluation antibacterial and antibiofilm activity of the antimicrobial peptide P34 against Staphylococcus aureus and Enterococcus faecalis. An Acad Bras Cienc. 2018;90:73-84.

27. Onsare JG, Arora DS. Antibiofilm potential of flavonoids extracted from Moringa oleifera seed coat against Staphylococcus aureus, Pseudomonas aeruginosa and Candida albicans. J Appl Microbiol. 2015;118:313-25.

28. Djordjevic D, Wiedmann M, McLandsborough LA. Microtiter plate assay for assessment of Listeria monocytogenes biofilm formation. Appl Environ Microbiol. 2002:68:2950-8.

29. Nikolic M, Vasic S, Durdevic J, Stefanovic O, Comic L. Antibacterial and antibiofilm activity of ginger (Zingiber officinale (roscoe)) ethanolic extract. Kragujevac J Sci. 2014;36:129-36.

30. Jadhav S, Shah R, Bhave M, Palombo AE. Inhibitory activity of yarrow essential oil on Listeria planktonic cells and biofilms. Food Control. 2013;29. 125-30.

31. Slama RB, Bekir K, Miladi H, Noumi A, Bakhrouf A. Adhesive ability and biofilm metabolic activity of Listeria monocytogenes strains before and after cold stress. Afr J Biotechnol. 2012;11:12475-82.

32. Wiegand I, Hilpert K, Hancock RE. Agar and broth dilution methods to determine the minimal inhibitory concentration (MIC) of antimicrobial substances. Nat Protoc. 2008;3:163.

33. Harput US, Arihan O, Iskit AB, Nagatsu A, Saracoglu I. Antinociceptive, free radical-scavenging, and cytotoxic activities of Acanthus hirsutus Boiss. J Med Food. 2011:14:767-74.

34. AL-Asady AAB, Ahmed NY, Mustafa TA. Cytotoxic and cytogenetic effects of aqueous and methanol crude extracts of Nicotiana tabacum on Rhabdomyosarcoma (RD) and L20B cell lines in vitro. Eur J Exp Biol. 2014;4:164-71.

35. Das MS, Devi G. In vitro cytotoxicity and glucose uptake activity of fruits of Terminalia belliricain Vero, L-6 and 3T3 cell lines. J Appl Pharm Sci. 2015;5: 092-5.

36. Hickman DL, Johnson SW. Evaluation of the aesthetics of physical methods of euthanasia of anesthetized rats. J Am Assoc Lab Anim Sci. 2011;50:695-701.

37. Dar RA, Shahnawaz M, Qazi PH. General overview of medicinal plants: a review. Aust J Pharm. 2017:6:349-51.

38. Cowan M. Plant products as antimicrobial agents. Clin Microbiol Rev. 1999; 12:564-82.

39. Sen A, Batra A. Determination of antimicrobial potentialities of different solvent extracts of the medicinal plant: Phyllanthusamarus Schum. and Thonn. Int J Green Pharm. 2012;6:50-6.

40. Devmurari VP. Phytochemical screening study and antibacterial evaluation of Symplocos racemosa Roxb. Arch Appl Sci Res. 2010;2:354-9.

41. Maitra S, Satardekar K. Pharmacological study of Symplocos racemosa Roxb. Int J Pharm Sci. 2017:9:106-13.
42. Shrestha P, Adhikari S, Lamichhane B, Shrestha BG. Phytochemical screening of the medicinal plants of Nepal. IOSR J Environ Sci Toxicol Food Technol. 2015;6:11-7.

43. Arora DS, Onsare JG. Antimicrobial potential of Moringa oleifera seed coat and its bioactive phytoconstituents. Korean J Microbiol Biotechnol. 2014b; 42:152-61.

44. Arora DS, Onsare JG. In vitro antimicrobial potential, biosafety and bioactive phytoconstituents of Moringa oleifera stem bark. World J Pharm Res. 2014C; 3:2772-88.

45. Bag A, Bhattacharyya SK, Pal NK, Chattopadhyay RR. In vitro antibacterial potential of Eugenia jambolana seed extracts against multidrug-resistant human bacterial pathogens. Microbiol Res. 2012;167:352-7.

46. Lin M, Lin Y, Lan C. Minimal inhibitory concentration (MIC) assay for Acinetobacter baumannii. Bio Protoc. 2014;4:e1308.

47. Mostafa AA, Al-Askar AA, Almaary KS, Dawoud TM, Sholkamy EN, Bakri MM. Antimicrobial activity of some plant extracts against bacterial strains causing food poisoning diseases. Saudi J Biol Sci. 2018;25:361-6.

48. Mathur R. Phytochemical and antimicrobial evaluation of plant extracts of Enicostem mahyssopifolium. J Pharmacogn Phytochem. 2013;2:30-6.

49. Jahan M, Abuhena M, Azad AK, Karim MM. In vitro antibacterial and antibiofilm activity of selected medicinal plants and spices extracts against multidrug resistant Pseudomonas aeruginosa. J Pharmacogn Phytochem. 2018;7:2114-21.

50. Slobodníkova L, Fialova S, Rendekova K, Kovac J, Mucaji P. Antibiofilm activity of plant polyphenols. Molecules. 2016;21:1717.

51. Vikram A, Jayaprakasha GK, Jesudhasan PR, Pillai SD, Patil BS. Suppression of bacterial cell-cell signalling, biofilm formation and type III secretion system by citrus flavonoids. J Appl Microbiol. 2010;109:515-27.

52. Lee JH, Kim YG, Cho HS, Ryu SY, Cho MH, Lee J. Coumarins reduce biofilm formation and the virulence of Escherichia coli 0157:H7. Phytomedicine. 2014;21:1037-42.

53. Harput-Hudaverdi US, Nagatsu A, Saracoglu I. Phytochemical and biological studies on Acanthus hirsutus Boiss. Planta Med. 2008:74:PB156.

54. Sidambaram RR, Dinesh MG, Jayalakshmi ET. An in vitro study of cytotoxic activity of Euphorbia hirta on Hep2 cells of human epithelioma of larynx. Int J Pharm Pharm Sci. 2011;3:3.

55. Kuete V, Omosa LK, Tala VR, Midiwo JO, Mbaveng AT, Swaleh S, Karaosmanoğlu O, Sivas H. Cytotoxicity of plumbagin, rapanone and 12 other naturally occurring quinones from Kenyan flora towards human carcinoma cells. BMC Pharmacol Toxicol. 2016;17:60.

56. Al-Marzoqi AH, Hameed IH, Idan SA. Analysis of bioactive chemical components of two medicinal plants (Coriandrum sativum and Melia azedarach) leaves using gas chromatography-mass spectrometry (GC-MS). Afr J Biotechnol. 2015;14:2812-30.

57. Priyanka C, Kumar P, Bankar SP, Karthik L. In vitro antibacterial activity and gas chromatography-mass spectroscopy analysis of Acacia karoo and Ziziphus mauritiana extracts. J Taibah Univ Sci. 2015;9:13-9.

58. Huang X, Ge SY, Liu JH, Wang Y, Liang XY, Yuan HB. Chemical composition and bioactivity of the essential oil from Artemisia lavandulaefolia (Asteraceae) on Plutella xylostella (Lepidoptera: Plutellidae). Fla Entomol. 2018;101:44-8

59. Parmar R, Kumar D. Study of chemical composition in wild edible mushroom Pleurotus cornucopiae (Paulet) from Himachal Pradesh, India by using Fourier transforms infrared spectrometry (FTIR), gas chromatographymass spectrometry (GCMS) and X-ray fluorescence (XRF). Biol Forum. 2015;7: 1057.

60. Gupta D, Kumar M. Evaluation of in vitro antimicrobial potential and GC-MS analysis of Camellia sinensis and Terminalia arjuna. Biotechnol Rep. 2017;13: 19-25.

61. Chandar B, Ramasamy MK. Evaluation of antioxidant, antibacterial activity of ethanolic extract in the leaves of Combretum albidum and gas chromatography-mass spectrometry analysis. Asian J Pharm Clin Res. 2016;9: 325-9.

\section{Publisher's Note}

Springer Nature remains neutral with regard to jurisdictional claims in published maps and institutional affiliations. 